\title{
EQUIDISTRIBUTION OF DIVERGENT ORBITS OF THE DIAGONAL GROUP IN THE SPACE OF LATTICES
}

\author{
OFIR DAVID AND URI SHAPIRA
}

\begin{abstract}
We consider divergent orbits of the group of diagonal matrices in the space of lattices in Euclidean space. We define two natural numerical invariants of such orbits: The discriminant an integer - and the type - an integer vector. We then study the question of the limit distributional behaviour of these orbits as the discriminant goes to infinity. Using entropy methods we prove that for divergent orbits of a specific type, virtually any sequence of orbits equidistribute as the discriminant goes to infinity. Using measure rigidity for higher rank diagonal actions we complement this result and show that in dimension 3 or higher only very few of these divergent orbits can spend all of their life-span in a given compact set before they diverge.
\end{abstract}

\section{INTRODUCTION}

One of the main points of interest in homogeneous dynamics is the study of $A$-orbits in the space of $n$-dimensional lattices $X=X_{n}:=\Gamma \backslash G$ where $G=\mathrm{SL}_{n}(\mathbb{R}), \Gamma=\mathrm{SL}_{n}(\mathbb{Z})$ and $A \leq \mathrm{SL}_{n}(\mathbb{R})$ is the subgroup of diagonal matrices with nonnegative entries. Many questions in this area can be formulated by defining a natural sequence of probability measures $\mu_{i}$ supported on orbits $x_{i} A$ (or a finite union of such orbits), and ask whether this sequence converges to some measure $\mu$, and in particular whether it equidistribute, namely $\mu=\mu_{\text {Haar }}$ is the Haar measure on $X$ (which is the unique $G$-invariant probability measure on $X$ ).

In this paper we focus on divergent orbits, namely orbits of the form $x A, x \in X$ where the map $A \rightarrow X$ defined by $a \mapsto x a$ is proper. Fixing a Haar measure on $A$, its push forward $\mu_{x A}$ to $x A$ is a well defined $A$-invariant locally finite measure. In particular, we observe that there is a unique $A$-invariant locally finite measure on $x A$ up to a scalar product. We will be interested in whether $\mu_{x_{i} A}$ (and averages of such measures) equidistribute for natural sequences of divergent orbits.

In the interest of defining equidistribution of locally finite measures, we introduce the following topology. Let $Z$ be a locally compact second countable Hausdorff space and let $\mathcal{M}(Z), \mathbb{P} \mathcal{M}(Z)$ denote the space of locally finite positive Borel measures on $Z$ and homothety classes of such (nonzero) measures respectively. For $\mu \in \mathcal{M}(Z)$ we let $[\mu]$ denote its homothety class. It is straightforward to define a topology on $\mathbb{P} \mathcal{M}(Z)$ such that for $\left[\mu_{n}\right],[\mu] \in \mathbb{P} \mathcal{M}(Z)$ we have the $\operatorname{limit} \lim \left[\mu_{n}\right]=[\mu]$ if and only if there exist constants $c_{n}$ such that for any compact set $K \subseteq Z,\left.\left.c_{n} \mu_{n}\right|_{K} \stackrel{w^{*}}{\longrightarrow} \mu\right|_{K}$, or equivalently for every $f, g \in C_{c}(Z)$ for which $\int g \mathrm{~d} \mu \neq 0$ we have $\frac{\int f \mathrm{~d} \mu_{n}}{\int g \mathrm{~d} \mu_{n}} \rightarrow \frac{\int f \mathrm{~d} \mu}{\int g \mathrm{~d} \mu}$. (see [6]).

We shall say that a sequence $0 \neq \mu_{n} \in \mathcal{M}(Z)$ equidistributes if $\left[\mu_{n}\right] \rightarrow\left[\mu_{\text {Haar }}\right]$.

We continue to describe the families of divergent orbits which are the focus of this paper. Recall first that we have the identification $X=\mathrm{SL}_{n}(\mathbb{Z}) \backslash \mathrm{SL}_{n}(\mathbb{R}) \cong \mathrm{PGL}_{n}(\mathbb{Z}) \backslash \mathrm{PGL}_{n}(\mathbb{R})$, i.e. we may consider general lattices up to homothety instead of unimodular lattices. We similarly consider orbits under the group of diagonal matrices in $\mathrm{PGL}_{n}(\mathbb{R})$. A finite index subgroup group of $\mathbb{Z}^{n}$ will be referred to as an integral lattice. Recall Mahler compactness criterion which says that a sequence of lattices $x_{i} \in X$ diverges if and only if the length of the shortest nonzero vector in $x_{i}$ converges 
to zero. It is then evident that integral lattices have divergent $A$-orbits because they contain a nonzero vector on each of the axes. On the other hand, it was shown in [7] that any divergent orbit contains an integral lattice. Evidently, each such orbit contains a unique integral lattice $L$ with minimal covolume. This lattice is characterized as the unique integral lattice in the orbit satisfying $\pi_{i}(L)=\mathbb{Z}$ for all $i$ where $\pi_{i}$ is the projection on the $i$-th coordinate. We call such an integral lattice axis primitive and its covolume is called the discriminant of the orbit. We note that if $L=\mathbb{Z}^{n} g$ for $g \in \operatorname{Mat}_{n}(\mathbb{Z})$, then $L$ is axis primitive if and only if each column of $g$ is a primitive vector, i.e. $\operatorname{gcd}\left(g_{1, i}, \ldots, g_{n, i}\right)=1$ for each $i$. We note that axis primitive is a stronger condition than just primitive which is an integral lattice $L \leq \mathbb{Z}^{n}$ such that there is no $0<c<1$ such that $c L$ is still contained in $\mathbb{Z}^{n}$, or equivalently if $L=\mathbb{Z}^{n} g$, then $\operatorname{gcd}\left\{g_{i, j} \mid 1 \leq i, j \leq n\right\}=1$.

A more refinement invariant of an integral lattice is its type. Given an integral lattice $L$, we define its type to be the finite abelian group $\mathbb{Z}^{n} / L$, which by the fundamental theorem of abelian groups can be characterized by a vector $\left(q_{1}, q_{2}, \ldots, q_{n}\right) \in \mathbb{N}^{n}$ where $q_{i} \mid q_{i+1}$ and $\mathbb{Z}^{n} / L \cong \mathbb{Z} / q_{1} \mathbb{Z} \times \cdots \times \mathbb{Z} / q_{n} \mathbb{Z}$. We define the type of a divergent orbit to be the type of its unique axis primitive integral lattice.

We shall focus on orbits of type $(1, q, \ldots, q)$. Any lattice $L$ of type $(1, q, \ldots, q)$ satisfies $q \mathbb{Z}^{n} \subseteq L$ and $L / q \mathbb{Z}^{n} \cong \mathbb{Z} / q \mathbb{Z}$, and if in addition $L$ is axis primitive, then we must have that $L=q \mathbb{Z}^{n}+\mathbb{Z}\left(1, p_{1}, \ldots, p_{n-1}\right)$ where $1 \leq p_{i} \leq q$ and $\left(p_{i}, q\right)=1$. This leads us to the following definition.

Definition 1.1. For $q \in \mathbb{N}$, we identify the sets $(\mathbb{Z} / q \mathbb{Z})^{\times}$and $\{1 \leq p \leq q \mid(p, q)=1\}$. With this identification in mind we set $\Lambda_{q}=\left\{\Gamma u_{\bar{p} / q} \mid \bar{p} \in\left((\mathbb{Z} / q \mathbb{Z})^{\times}\right)^{n-1}\right\}$ where $u_{\bar{x}}=\left(\begin{array}{cc}1 & \bar{x} \\ 0_{(n-1) \times 1} & I_{(n-1) \times(n-1)}\end{array}\right) \in$ $\mathrm{SL}_{n}(\mathbb{R})$ for $\bar{x} \in \mathbb{R}^{n-1}$, and identify it in the natural way with the sets $\left((\mathbb{Z} / q \mathbb{Z})^{\times}\right)^{n-1},\{1 \leq p \leq q \mid(p, q)=1\}^{n-1}$ and $\left\{u_{\bar{p} / q} \mid \bar{p} \in\left((\mathbb{Z} / q \mathbb{Z})^{\times}\right)^{n-1}\right\}$.

Thus, the orbits of type $(1, q, \ldots, q)$ are in one to one correspondence with $\Lambda_{q}$. Note in particular that we have $\left|\Lambda_{q}\right|=\varphi(q)^{n-1}$. With these notations we shall establish the following result which generalizes the main result in [1].

Theorem 1.2. The following is true:

(1) There exist $\Lambda_{q}^{\prime} \subseteq \Lambda_{q}$ with $\lim _{q \rightarrow \infty} \frac{\left|\Lambda_{q}^{\prime}\right|}{\left|\Lambda_{q}\right|}=1$, s.t. given $x_{q} \in \Lambda_{q}^{\prime}$ we have that $\left[\mu_{x_{q} A}\right] \rightarrow\left[\mu_{\text {Haar }}\right]$.

(2) If $0<\alpha \leq 1$ and $\Lambda_{q}^{\prime} \subseteq \Lambda_{q}$ such that $\left|\Lambda_{q}^{\prime}\right| \geq \alpha\left|\Lambda_{q}\right|$, then $\left[\sum_{x \in \Lambda_{q}^{\prime}} \mu_{x A}\right] \rightarrow\left[\mu_{\text {Haar }}\right]$.

(3) We have the convergence $\left[\sum_{x \in \Lambda_{q}} \mu_{x A}\right] \rightarrow\left[\mu_{\text {Haar }}\right]$.

Remark 1.3. While the implications $(1) \Rightarrow(2) \Rightarrow(3)$ are easy, we actually first prove (3) and show that $(3) \Rightarrow(2) \Rightarrow(1)$ using the fact that $\mu_{\text {Haar }}$ is ergodic (with respect to the action of non-trivial elements of $A$ ), hence an extreme point in the set of $A$-invariant probability measures.

Recall that by Mahler criterion, compact sets in $X$ are closed sets $K$ such that inf $\{\|v\| \mid 0 \neq v \in L, L \in K\}>$ 0 . Given a rational lattice $x \in X$, its $A$-orbit diverges since $x$ contains an element in each axis and at least one of them contains a very short vector once we apply an element of $A$ of large norm. This motivates us to truncate the orbit and focus on its part where all non-zero vectors on the axis are of length $\geq 1$. Recall that $A$ is naturally isomorphic to $\mathbb{R}_{0}^{n}=\left\{\bar{t} \in \mathbb{R}^{n} \mid \sum_{1}^{n} t_{i}=0\right\}$ via the map 
$\bar{t} \mapsto \operatorname{diag}\left(e^{t_{1}}, \ldots, e^{t_{n}}\right)$. Moreover, for $x \in \Lambda_{q}$, identifying $A$ with the divergent orbit $x A$ under $a \mapsto x a$, it is easily verified (see section 2 for details) that the truncated part of the orbit mentioned above is $\Delta_{\text {full }, q}=\ln (q) \Delta_{\text {full }}$ where

$$
\Delta_{\text {full }}=\left\{\bar{t} \in \mathbb{R}_{0}^{n} \mid t_{i} \geq 0 \geq t_{1} \geq-1, i \geq 2\right\} .
$$

As $a \mapsto x a$ diverges quickly when $a \notin \Delta_{f u l l, q}$, we can expect that if $\left[\mu_{x A}\right]$ is close $\left[\mu_{\text {Haar }}\right]$, then its restriction to $x \Delta_{\text {full, } q}$ must also be close to $\mu_{\text {Haar }}$ up to some normalization (see Corollary 2.3 for the details). By this argument and Theorem 1.2 we expect that for most $x \in \Lambda_{q}$, the truncated orbit $x \Delta_{f u l l, q}$ would be roughly equidistributed in $X$. In particular, fixing some compact set $K \subseteq X$ there should be very few $x \in \Lambda_{q}$ such that $x \Delta_{f u l l, q} \subseteq K$. Indeed, using classification of $A$-invariant measures with positive entropy (see [2]) we establish the following result.

Theorem 1.4. Let $n \geq 3$. Fix a compact set $K \subseteq X_{n}$ and let $\Lambda_{q, K}=\left\{x \in \Lambda_{q} \mid x \cdot \Delta_{\text {full, } q} \subseteq K\right\}$. Then for any $\varepsilon>0$ we have that $\left|\Lambda_{q, K}\right|=O\left(\left|\Lambda_{q}\right|^{\varepsilon}\right)$.

1.1. Continued fraction expansion. In the case of $n=2$, there is a well known connection between the $A$-orbits in $X_{2}$ and continued fraction expansions (c.f.e) of numbers in $[0,1]$. This connection was capitalized by the authors in [1]. We recall the interpretation of the results above in the realm of c.f.e in order to give some more intuition to them.

Recall that the Gauss map $T:(0,1] \rightarrow[0,1]$ is defined by $T(x)=\frac{1}{x}-\left\lfloor\frac{1}{x}\right\rfloor$ and the Gauss-Kuzmin measure $\nu_{\text {Gauss }}$ on $[0,1]$ is defined by $\nu_{\text {Gauss }}(f):=\frac{1}{\ln (2)} \int_{0}^{1} f(t) \frac{1}{1+t} \mathrm{dt}$. It is well known that $\nu_{\text {Gauss }}$ is a $T$-invariant ergodic probability measure. Hence, by the pointwise ergodic theorem, for almost every $x \in[0,1]$, the sequence $\frac{1}{N} \sum_{0}^{N-1} \delta_{T^{i}(x)}$ equidistribute, i.e. it converges to $\nu_{\text {Gauss }}$.

This claim is certainly not true for all $x$. Indeed, if $\frac{p}{q} \in \mathbb{Q}$ is rational, then it has a finite c.f.e of length len $\left(\frac{p}{q}\right) \in \mathbb{N}$ so that $T^{\operatorname{len}(p / q)}\left(\frac{p}{q}\right)=0$, and in particular $T^{\operatorname{len}(p / q)+1}\left(\frac{p}{q}\right)$ is not defined. In this $\operatorname{len}(p / q)-1$

case we may still ask whether $\nu_{p / q}:=\frac{1}{\operatorname{len}(p / q)} \sum_{0}^{\operatorname{lon}(p / q)} \delta_{T^{i}(p / q)}$ converge to $\nu_{\text {Gauss }}$ for a well chosen sequence of rationals.

The interpretation of Theorem 1.2 in this language is that there exist $\Lambda_{q}^{\prime} \subseteq(\mathbb{Z} / q \mathbb{Z})^{\times}$such that $\lim _{q \rightarrow \infty} \frac{\left|\Lambda_{q}^{\prime}\right|}{\varphi(q)}=1$ and for any sequence $p_{q} \in \Lambda_{q}^{\prime}$ we have that $\nu_{p_{q} / q} \rightarrow \nu_{\text {Gauss }}$ as $q \rightarrow \infty$.

Theorem 1.4 is not applicable for $n=2$ since the classification of measures with positive entropy only holds for $n>2$. On the other hand, the argument of maximal entropy still holds and leads to the following result in [1]. For any fixed $M$, let

$$
\Lambda_{q, M}=\left\{p \in(\mathbb{Z} / q \mathbb{Z})^{\times} \mid \frac{p}{q}=\left[0 ; x_{1}, \ldots, x_{l e n(p / q)}\right], x_{i} \leq M\right\} .
$$

Then there exists some $\varepsilon=\varepsilon(M)>0$ such that $\left|\Lambda_{q, M}\right|=O\left(\left|\Lambda_{q}\right|^{1-\varepsilon}\right)$. There is no known lower bound for the size of these sets, and in particular the claim that these sets are non empty (for some fixed $M$ ) is known as Zaremba's conjecture.

1.2. Equidistribution over the adeles. Another conceptual viewpoint of these results is achieved by lifting the discussion to the adeles.

Let $\mathbb{A}$ denote the ring of adeles over $\mathbb{Q}$ and consider the space $X_{\mathbb{A}}:=\Gamma_{\mathbb{A}} \backslash G_{\mathbb{A}}$, where $G_{\mathbb{A}}=\mathrm{PGL}_{n}(\mathbb{A})$ and $\Gamma_{\mathbb{A}}=\mathrm{PGL}_{n}(\mathbb{Q})$. Let $A_{\mathbb{A}} \leq G_{\mathbb{A}}$ denote the subgroup of diagonal matrices. Note that the orbit $\tilde{x}_{0} A_{\mathbb{A}}$ is a closed orbit (where $\tilde{x}_{0}$ denotes the identity coset $\Gamma_{\mathbb{A}}$ ). In particular, fixing once and for all 
a Haar measure on $A_{\mathbb{A}}$ we obtain a Haar measure on the quotient $s t a b_{A_{\mathbb{A}}}\left(\tilde{x}_{0}\right) \backslash A_{\mathbb{A}}$ and by pushing the latter into $X_{\mathbb{A}}$ via the proper embedding induced by the map $a \mapsto \tilde{x}_{0} a$, we obtain an $A_{\mathbb{A}}$-invariant locally finite measure $\mu_{\tilde{x}_{0} A_{\AA}}$ supported on the closed orbit $\tilde{x}_{0} A_{\AA}$.

Letting $\bar{u}_{\bar{p} / q}=\left(I, u_{\bar{p} / q}, u_{\bar{p} / q}, \ldots\right) \in G_{\mathbb{A}}$ for $\bar{p} \in \Lambda_{q}$, the orbit $\tilde{x} A_{\mathbb{A}} \bar{u}_{\bar{p} / q}^{-1}$ is mapped under the natural natural epimorphism $\Gamma_{\mathbb{A}} \backslash G_{\mathbb{A}} \rightarrow \Gamma \backslash G$ to the union of orbits $\bigcup_{x \in \Lambda_{q}} x A$. One can obtain the following result which easily seen to imply Theorem 1.2 and actually these claims are equivalent.

Theorem 1.5. For any choice of $\bar{p}^{(q)} \in \Lambda_{q}$ we have that $\left[\bar{u}_{\bar{p}^{(q)} / q} \mu_{\tilde{x}_{0} A_{\mathbb{A}}}\right] \rightarrow\left[\mu_{\mathbb{A}, H a a r}\right]$ as $q \rightarrow \infty$.

A similar result was deduced in [1] from the analogue of Theorem 1.2 for the $n=2$ case. Since the proofs are identical we do not repeat the details here.

We believe that Theorem 1.5 should be true for more general sequences of push-forwards. In particular for the case $n=2$, Theorem 1.5 is equivalent to the following statement (see [1]): Given any $g_{i} \in G_{\mathbb{A}}$ such that (1) the real component of $g_{i}$ is the identity and (2) the projection to $G_{\mathbb{A}} / A_{\mathbb{A}}$ diverges, then we have the convergence in homothety $\left[g_{i} \cdot \mu_{\tilde{x}_{0} A_{\mathbb{A}}}\right] \rightarrow\left[\mu_{\mathbb{A}, \text { Haar }}\right]$. We believe that some version of this statement should be true in higher dimension, though there should be further conditions on $g_{i}$, for example in order for $g_{i} \cdot \mu_{\tilde{x}_{0} A_{\mathbb{A}}}$ not to get "stuck" in a subspace.

1.3. Acknowledgment. The authors would like to thank Elon Lindenstrauss for valuable discussions and acknowledge the support of ISF 357/13.

\section{Axis lattices and truncated $A$-Orbits}

In this paper we will be interested in the orbits of lattices of the form $\mathbb{Z}^{n} u_{\bar{p} / q}$ where $\bar{p} \in \Lambda_{q}$ (see Definition 1.1). These lattices contain a vector on each axis, therefore the map $a \mapsto \mathbb{Z}^{n} u_{\bar{p} / q} a$ from $A$ to $X$ is proper, and we say that the orbit diverges. We begin with a slightly more generalized discussion to understand this phenomenon.

For $\bar{t} \in \mathbb{R}_{0}^{n}$ we denote by $a(\bar{t})$ the diagonal matrix $\operatorname{diag}\left(e^{t_{1}}, \ldots, e^{t_{n}}\right) \in A$ and set $a_{q}(\bar{t}):=$ $a(\ln (q) \bar{t})=\operatorname{diag}\left(q^{t_{1}}, \ldots, q^{t_{n}}\right)$. When there is no ambiguity, we shall identify between $\mathbb{R}_{0}^{n}$ and the group $A$ of positive diagonal matrices. For the rest of the paper we fix a Haar measure $\lambda$ on $A$.

Definition 2.1. Let $L \subseteq \mathbb{R}^{n}$ be a unimodular lattice.

- The lattice $L$ is called an axis lattice if $L \cap \mathbb{R} e_{i} \neq 0,1 \leq i \leq n$.

- For $1 \leq i \leq n$ we write $\operatorname{covol}(L, i):=\operatorname{vol}\left(\mathbb{R} e_{i} /\left(L \cap \mathbb{R} e_{i}\right)\right)$ (which is finite for axis lattices).

- Given an axis lattice we define

$$
\begin{aligned}
A_{L} & =\left\{\bar{t} \in \mathbb{R}_{0}^{n} \mid \operatorname{covol}(\operatorname{La}(\bar{t}), i) \geq 1 \forall i \in\{1, \ldots, n\}\right\} \\
& =\left\{\bar{t} \in \mathbb{R}_{0}^{n} \mid t_{i} \geq-\ln (\operatorname{covol}(L, i)) \forall i \in\{1, \ldots, n\}\right\} .
\end{aligned}
$$

- We shall write $\tau_{L}=\frac{1}{n} \sum_{1}^{n} \ln (\operatorname{covol}(L, i))$.

We note that any axis lattice $x \in X$ contains in its $A$-orbit an axis lattice $x^{\prime}$ such that $\operatorname{covol}\left(x^{\prime}, i\right)=$ $\operatorname{covol}\left(x^{\prime}, j\right)$ for all $i, j$ and $A_{x^{\prime}}=\left\{\bar{t} \in \mathbb{R}_{0}^{n} \mid t_{i} \geq-\tau_{x}\right\}$. It then follows that $\frac{\lambda\left(A_{x}\right)}{\tau_{x}^{(n-1)}}=\frac{\lambda\left(A_{x^{\prime}}\right)}{\tau_{x}^{(n-1)}}=$ $\lambda\left(\left\{\bar{t} \in \mathbb{R}_{0}^{n} \mid t_{i} \geq-1\right\}\right)$ is constant.

Recall that for a divergent orbit $x A$ we let $\mu_{x A}$ be the locally finite $A$-invariant measure on $x A$ (which is the pushforward of the Haar measure $\lambda$ on $A$ ). By [7] the orbit $x A$ contains a rational lattice, so in particular $x$ must be an axis lattice. We denote by $\mu_{x}$ the probability normalization of the restriction of $\mu_{x A}$ to $x \cdot A_{x}$. 
EQUIDISTRIBUTION OF DIVERGENT ORBITS OF THE DIAGONAL GROUP IN THE SPACE OF LATTICES 5

For a lattice $L$ we set $h t(L)=\max \left\{\|v\|_{\infty}^{-1} \mid 0 \neq v \in L\right\}$ and define $X \leq M=\{x \in X \mid h t(x) \leq M\}$ (and similarly we define $X^{<M}, X^{\geq M}$ and $X^{>M}$ ).

The following lemma tells us that when investigating the orbit $x A$, one may focus only on $x A_{x}$.

Lemma 2.2. Let $f \in C_{c}(X)$ with $\operatorname{supp}(f) \subseteq X^{\leq M}$ and $x \in X$ with $\tau_{x} \geq M$. Then

$$
\left|\left(\lambda\left(A_{x}\right)^{-1} \mu_{x A}-\mu_{x}\right)(f)\right| \leq n \cdot 2^{n} \frac{M}{\tau_{x}}\|f\| .
$$

Proof. Up to choosing a different representative of the $A$-orbit, we may assume that $A_{x}=\left\{\bar{t} \in \mathbb{R}_{0}^{n} \mid \min t_{i} \geq-\tau_{x}\right\}$. Setting $A_{x, M}=\left(1+\frac{M}{\tau_{x}}\right) A_{x}$ we obtain that $h t(x a(\bar{t}))>M$ for every $\bar{t} \in \mathbb{R}_{0}^{n} \backslash A_{x, M}$. We conclude that

$$
\begin{aligned}
\left|\left(\lambda\left(A_{x}\right)^{-1} \mu_{x A}-\mu_{x}\right)(f)\right| & =\left|\left(\lambda\left(A_{x}\right)^{-1} \mu_{x A}||_{x\left(A_{x, M} \cap A_{x}^{c}\right)}\right)(f)\right| \leq\left(\frac{\lambda\left(A_{x, M}\right)}{\lambda\left(A_{x}\right)}-1\right)\|f\| \\
& =\left[\left(1+\frac{M}{\tau_{x}}\right)^{(n-1)}-1\right]\|f\| \leq\left[(n-1)\left(1+\frac{M}{\tau_{x}}\right)^{(n-2)} \frac{M}{\tau_{x}}\right]\|f\| \leq n \cdot 2^{n} \frac{M}{\tau_{x}}\|f\| .
\end{aligned}
$$

From this we conclude the following corollary which says that taking the limit of the locally finite $A$-invariant measures and their corresponding restrictions is basically equivalent.

Corollary 2.3. Let $\mathcal{F}_{i}=\left\{x_{i, 1}, \ldots, x_{i, m(i)}\right\}$ be a family of axis lattices such that $\tau_{i}=\tau_{x_{i, j}}$ is independent of $1 \leq j \leq m(i)$ and $\tau_{i} \rightarrow \infty$. Then $\frac{1}{\left|\mathcal{F}_{i}\right|} \sum_{j} \mu_{x_{i, j}} \stackrel{w^{*}}{\longrightarrow} \mu$ implies that $\left[\sum_{j} \mu_{x_{i, j} A}\right] \rightarrow[\mu]$.

This corollary allow us to prove Theorem 1.2 by proving its analogue with the probability measures $\mu_{x}$ instead of the locally finite measures $\mu_{x A}$.

The measures $\frac{1}{\left|\mathcal{F}_{i}\right|} \sum_{j} \mu_{x_{i, j}}$ in the corollary above are averages over the elements in $\mathcal{F}_{i}$, and each $\mu_{x}$ is an average (integral) over part of the orbit. To study these notions we use the following notations.

Definition 2.4. Let $\mu$ be a probability measure on $X, \nu$ a probability measure on $\mathbb{R}_{0}^{n}$ and let $\Lambda \subseteq X$ be a finite subset.

(1) We define $\delta_{\Lambda}=\frac{1}{|\Lambda|} \sum_{x \in \Lambda} \delta_{x}$ where $\delta_{x}$ is the Dirac measure at $x$.

(2) For $\Omega \subseteq \mathbb{R}_{0}^{n}$ with positive Lebesgue measure define $\mu^{\Omega}=\frac{1}{\lambda(\Omega)} \int_{\Omega}(a(-\bar{t}) \mu) \mathrm{d} \lambda(\bar{t})$.

Using these notations, if $x$ is an axis lattice, then $\mu_{x}=\delta_{x}^{A_{x}}$. If $x \in \Lambda_{q}$, then it is easily verified that $A_{x}=\ln (q) \Delta_{f u l l}$, so in particular $A_{x}$ is only a function of $q$. It follows that $\frac{1}{\left|\Lambda_{q}\right|} \sum_{x \in \Lambda_{q}} \mu_{x}=\delta_{\Lambda_{q}}^{\ln (q) \Delta_{f u l l}}$, so in order to prove Theorem 1.2 part (3), it is enough to prove that $\delta_{\Lambda_{q}}^{\ln (q) \Delta_{\text {full }}} \rightarrow \mu_{\text {Haar }}$.

The permutation group $S_{n}$ acts naturally on lattices in $X_{n}$ and on elements from $A \cong \mathbb{R}_{0}^{n}$. Moreover, for $x \in X_{n}, a \in A$ and $\sigma \in S_{n}$ we have that $\sigma(x a)=\sigma(x) \sigma(a)$. In the next lemma we show that this action restricts to an action on $\Lambda_{q} \Delta_{f u l l, q}$. In particular, for $\sigma=(1, \ldots, n)$ the fundamental domain is $\Lambda_{q} \Delta_{q}$ where $\Delta_{q}=\ln (q) \Delta$ and

$$
\Delta=\left\{\bar{t} \in \mathbb{R}_{0}^{d+1} \mid t_{i} \geq 0 \geq t_{1} \geq-1+t_{i}, \forall i \geq 2\right\},
$$

which allows us to consider in our computations the measure $\delta_{\Lambda_{q}}^{\ln (q) \Delta}$ instead of $\delta_{\Lambda_{q}}^{\ln (q) \Delta_{\text {full }}}$ (see figure 2.1). 


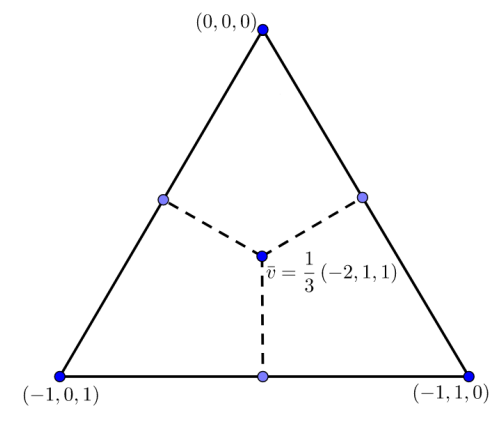

Figure 2.1. For $d=2$, the whole triangle is $\Delta_{\text {full }}$, while the upper third part is $\Delta$.

Lemma 2.5. For any $q$ we have that $\delta_{\Lambda_{q}}^{\ln (q) \Delta_{\text {full }}}=\frac{1}{n} \sum_{i=1}^{n} \sigma_{n}^{i}\left(\delta_{\Lambda_{q}}^{\ln (q) \Delta}\right)$ where $\sigma_{n}=(1, \ldots, n) \in S_{n}$.

Proof. In order to make the problem more symmetric we consider the set $\tilde{\Lambda}_{q}=\Lambda_{q} a_{q}\left(\frac{1}{n}(1-n, 1, \ldots, 1)\right)$. Thus the lattices $\tilde{x} \in \tilde{\Lambda}_{q}$ are exactly those with basis of the form $\left\{q^{1 / n} e_{2}, \ldots, q^{1 / n} e_{n}, q^{1 / n} \cdot \frac{1}{q}\left(e_{1}+\sum_{j>1} p_{j} e_{j}\right)\right\}$ where $p_{j} \in(\mathbb{Z} / q \mathbb{Z})^{\times}$. Equivalently these are lattices generated by $q^{1 / n} \mathbb{Z}^{n}$ and a vector $q^{\frac{1-n}{n}} \sum_{1}^{n} p_{i} e_{i}$ where $p_{i} \in(\mathbb{Z} / q \mathbb{Z})^{\times}$. It follows that for $\tilde{x} \in \tilde{\Lambda}_{q}$ we have that $A_{\tilde{x}}=\tilde{\Delta}_{f u l l, q}=\ln (q) \tilde{\Delta}_{\text {full }}$ where $\tilde{\Delta}_{\text {full }}=\left\{\bar{t} \in \mathbb{R}_{0}^{n} \mid t_{i} \geq-\frac{1}{n}\right\}$.

Clearly, the sets $\tilde{\Lambda}_{q}$ and $\tilde{\Delta}_{f u l l}$ is stable under $S_{n}$ and hence also $\tilde{\Lambda}_{q} \tilde{\Delta}_{f u l l, q}=\Lambda_{q} \Delta_{f u l l, q}$. If $H \leq S_{n}$ and $\tilde{F}$ is a fundamental domain of $H$ in $\tilde{\Delta}_{f u l l}$, then $\tilde{\Lambda}_{q} F$ is a fundamental of $H$ in $\tilde{\Lambda}_{q} \tilde{\Delta}_{f u l l, q}$. Using the fact that $H$ permutes the elements in $\tilde{\Lambda}_{q}$ we obtain that therefore

$$
\delta_{\Lambda_{q}}^{\ln (q) \Delta_{\text {full }}}=\delta_{\tilde{\Lambda}_{q}}^{\ln (q) \tilde{\Delta}_{\text {full }}}=\frac{1}{|H|} \sum_{h \in H} \delta_{\tilde{\Lambda}_{q}}^{\ln (q) h(F)}=\frac{1}{|H|} \sum_{h \in H} h\left(\delta_{\tilde{\Lambda}_{q}}^{\ln (q) F}\right) .
$$

Carrying this argument back to $\Lambda_{q}$ we need to take the fundamental domain $F=\tilde{F}+\frac{1}{n}(1-n, 1, \ldots, 1)$ instead and obtain $\delta_{\Lambda_{q}}^{\ln (q) \Delta_{\text {full }}}=\frac{1}{|H|} \sum_{h \in H} h\left(\delta_{\Lambda_{q}}^{\ln (q) F}\right)$. If $H=\langle(1, \ldots, n)\rangle$, then we can choose $\tilde{F}=$ $\left\{\bar{t} \in \mathbb{R}_{0}^{n} \mid t_{1} \geq t_{i} \geq-\frac{1}{n}, \forall i\right\}$ and then $F=\Delta$, thus completing the proof.

Corollary 2.6. If $\delta_{\Lambda_{q}}^{\ln (q) \Delta} \stackrel{w^{*}}{\longrightarrow} \mu_{\text {Haar }}$, then $\delta_{\Lambda_{q}}^{\ln (q) \Delta_{\text {full }}} \stackrel{w^{*}}{\longrightarrow} \mu_{\text {Haar }}$.

Proof. Let $\sigma_{n}=(1, \ldots, n) \in S_{n}$ and set $P_{\sigma_{n}}=\sum E_{\sigma_{n}(i), i}$ be the corresponding permutation matrix (where $E_{i, j}$ is the zero matrix with 1 in the $(i, j)$ coordinate). Let $g=P_{\sigma_{n}}$ if $n$ is odd and $g=$ $\operatorname{diag}(-1,1, \ldots, 1) P_{\sigma_{n}}$ if $n$ is even, so in any way we have that $g \in \mathrm{SL}_{n}(\mathbb{R})$ and $g(x)=P_{\sigma_{n}}(x)=h(x)$ for any $x \in X_{n}$.

By Lemma 2.5, we have that $\delta_{\Lambda_{q}}^{\ln (q) \Delta_{f u l l}}=\frac{1}{n} \sum_{i=1}^{n} \sigma_{n}^{i}\left(\delta_{\Lambda_{q}}^{\ln (q) \Delta}\right)$. By the assumption $\delta_{\Lambda_{q}}^{\ln (q) \Delta} \stackrel{w^{*}}{\longrightarrow} \mu_{\text {Haar }}$ which is $G$-invariant, hence $\delta_{\Lambda_{q}}^{\ln (q) \Delta_{\text {full }}}=\frac{1}{n} \sum_{i=1}^{n} \sigma_{n}^{i}\left(\delta_{\Lambda_{q}}^{\ln (q) \Delta}\right)=\frac{1}{n} \sum_{i=1}^{n} g^{i}\left(\delta_{\Lambda_{q}}^{\ln (q) \Delta}\right) \stackrel{w^{*}}{\longrightarrow} \mu_{\text {Haar }}$. 


\section{Proofs of the MAIN Results}

Once we restrict our attention to $\Lambda_{q} \cdot \Delta$, the flow induced by the action of the line spanned by $\frac{1}{n}(1-n, 1, \ldots, 1)$, which expands "uniformly" the set $\Lambda_{q}$, will play an important role in our analysis.

Definition 3.1. We set $\vec{v}=\frac{1}{n}(1-n, 1, \ldots, 1)$. Let $E(\Delta)=\left\{(\bar{w}, r) \in \Delta \times \mathbb{R}_{>0} \mid \bar{w}+r \cdot \vec{v} \in \Delta\right\}$, i.e. the intervals in the direction $\vec{v}$ inside $\Delta$. For $(\bar{w}, r) \in E(\Delta)$ and a probability measure $\mu$ on $X$ we define $\mu^{[\bar{w}, r, q]}=\frac{1}{r} \int_{0}^{r} a_{q}(-(\bar{w}+r \overrightarrow{\mathrm{v}})) \mu \mathrm{dt}$.

We note that for $x \in X$, taking $\mu=\delta_{x}$, the measure $\delta_{x}^{[\bar{w}, r, q]}$ is the uniform measure on the $\left\{x a_{q}(\bar{w}+r \overrightarrow{\mathrm{v}})\right\}$ as a part of the orbit $x a_{q}(\bar{w}) a_{q}(\mathbb{R} \overrightarrow{\mathrm{v}})$. The main idea of the proof is that given a partial weak star limit $\mu$ of some sequence $\delta_{\Lambda_{q}^{\prime}}^{[\bar{w}, r, q]}$, which is $a_{q}(\overrightarrow{\mathrm{v}})$ invariant, we can give a lower bound on the entropy of $\mu$ by giving a lower bound for the entropy of $\delta_{\Lambda_{q}^{\prime}}^{[\bar{w}, r, q]}$ for suitable choices of partitions.

3.1. Entropy lower bound on measure averages. We begin by recalling the definition of entropy and the uniqueness of measures of maximal entropy in our setting. For more details the reader is referred to $[5,8]$.

Definition 3.2. Let $Y$ be any measurable space, $S: Y \rightarrow Y$ a measurable function, $\mu$ a probability measure on $Y$ and $\mathcal{P}=\left\{P_{i}\right\}_{1}^{N}$ a finite measurable partition of $Y$. Then

- The entropy of $\mu$ with respect to $\mathcal{P}$ is $H_{\mu}(\mathcal{P})=-\sum \mu\left(P_{i}\right) \ln \left(\mu\left(P_{i}\right)\right)$.

- For $m_{1}<m_{2}$ integers we write $\mathcal{P}_{m_{1}}^{m_{2}}=\bigvee_{m_{1}}^{m-1} S^{-i} \mathcal{P}$, and $\mathcal{P}^{m}:=\mathcal{P}_{0}^{m}$.

- If $\mu$ is $S$-invariant, we set $h_{\mu}(S, \mathcal{P})=\lim _{m \rightarrow \infty} \frac{1}{m} H_{\mu}\left(\mathcal{P}^{m}\right)$ and $h_{\mu}(S)=\sup _{\mathcal{P}} h_{\mu}(S, P)$.

In the setting of $X_{n}=\mathrm{SL}_{n}(\mathbb{Z}) \backslash \mathrm{SL}_{n}(\mathbb{R})$ we will always use the map $T(x)=x a_{q}(\vec{v})$, in which case there is a unique $T$-invariant measure of maximal entropy. The next theorem is a combination of Theorems 7.6, 7.9 in [3] and the fact that $G$ is generated by $U, U^{t r}$.

Theorem 3.3 (see [3]). Let $\mu$ be a T-invariant probability measure on $X$. Then $h_{\mu}(T) \leq h_{\mu_{\text {Haar }}}(T)=$ $d$, and there is equality if and only if $\mu=\mu_{\text {Haar }}$.

For $\Lambda \subseteq X$ finite and $(\bar{w}, r) \in E(\Delta)$, the measure $\delta_{\Lambda}^{[\bar{w}, r, q]}$ is a double average of measures, once for the points in $\Lambda$ and once in the direction in which we compute the entropy. The next lemma provides a lower bound on the entropy for such averages.

Lemma 3.4. Let $Y$ be any measurable space, let $S: Y \rightarrow Y$ be some measurable function and $\mathcal{P}$ a partition of $Y$. For a probability measure $\mu$ on $Y$ we write $\mu^{k}=\frac{1}{k} \sum_{i=0}^{k-1} S^{i} \mu$. Then

(1) If $(\Omega, \mathrm{dx})$ is a measurable space, $x \mapsto \nu_{x}$ is a measurable map from $\Omega$ to the probability measures on $Y$ and $\nu=\int_{\Omega} \nu_{x} \mathrm{dx}$, then

$$
H_{\nu}(\mathcal{P}) \geq \int_{\Omega} H_{\nu_{x}}(\mathcal{P}) \mathrm{dx}
$$

(2) For $m \leq k$ we have that

$$
\frac{1}{m} H_{\mu^{k}}\left(\mathcal{P}^{m}\right) \geq \frac{1}{k} H_{\mu}\left(\mathcal{P}^{n}\right)-\frac{m}{k} \ln |\mathcal{P}|
$$

Proof. (1) Since the function $\alpha: x \mapsto-x \ln (x)$ is concave in $[0,1]$, we obtain that

$$
H_{\nu}(\mathcal{P})=\sum_{\mathcal{Q} \in \mathcal{P}} \alpha(\nu(\mathcal{Q}))=\sum_{\mathcal{Q} \in \mathcal{P}} \alpha\left(\int \nu_{x}(\mathcal{Q}) \mathrm{dx}\right) \geq \sum_{\mathcal{Q} \in \mathcal{P}} \int \alpha\left(\nu_{x}(\mathcal{Q})\right) \mathrm{dx}=\int H_{\nu_{x}}(\mathcal{P}) \mathrm{dx} .
$$


(2) Write $k=l m+r \leq m(l+1)$ where $0 \leq r<m$, and using subadditivity we get that for $0 \leq u \leq m-1$ we have

$$
\begin{aligned}
H_{\mu}\left(\mathcal{P}^{k}\right) & \leq H_{\mu}\left(\mathcal{P}^{l m+r}\right) \\
& \leq \sum_{i=0}^{u-1} H_{\mu}\left(T^{-i} \mathcal{P}\right)+\sum_{v=0}^{l-1} H_{\mu}\left(\mathcal{P}_{v m+u}^{(v+1) m+u}\right)+\sum_{i=l m+u}^{l m+m-1} H_{\mu}\left(T^{-i} \mathcal{P}\right) \\
& \leq m \log |\mathcal{P}|+\sum_{v=0}^{l-1} H_{T^{v m+u} \mu}\left(\mathcal{P}^{m}\right) .
\end{aligned}
$$

Summing over $0 \leq u \leq m-1$ we get that

$$
\begin{aligned}
m H_{\mu}\left(\mathcal{P}^{k}\right)-m^{2} \ln |\mathcal{P}| & \leq \sum_{u=0}^{m-1} \sum_{v=0}^{l-1} H_{\left(T^{v m+u} \mu\right)}\left(\mathcal{P}^{m}\right)=\sum_{j=0}^{l m-1} H_{\left(T^{j} \mu\right)}\left(\mathcal{P}_{m}\right) \\
& \leq \sum_{j=0}^{k-1} H_{\left(T^{j} \mu\right)}\left(\mathcal{P}^{m}\right) \leq k H_{\mu^{k}}\left(\mathcal{P}^{m}\right)
\end{aligned}
$$

where in the last step we used part (1). It follows that $\frac{1}{m} H_{\mu^{k}}\left(\mathcal{P}^{m}\right) \geq \frac{1}{k} H_{\mu}\left(\mathcal{P}^{k}\right)-\frac{m}{k} \ln |\mathcal{P}|$.

3.2. Small balls have small measure. The second part of Lemma 3.4 should be thought of as instead of taking the average of the measure $\mu$ along the $T$-direction, we take a greater refinement of the partition $\mathcal{P}$. Thus, the atoms in $\mathcal{P}^{k}$ become smaller and we would expect them to have small $\mu$ measure, which would provide a lower bound on the entropy since

$$
H_{\mu}\left(\mathcal{P}^{k}\right)=-\sum_{P \in \mathcal{P}^{k}} \mu(P) \ln (\mu(P)) \geq-\sum_{P \in \mathcal{P}^{k}} \mu(P)\left[\max _{Q \in \mathcal{P}^{k}} \ln (\mu(Q))\right]=-\max _{Q \in \mathcal{P}^{k}} \ln (\mu(Q)) .
$$

The main problem in this argument is that the space $X$ is not compact, hence $\mathcal{P}$ has a noncompact atom, and therefore not all of the atoms in $\mathcal{P}^{k}$ are small. After defining what "small" is in our context, we salvage this argument in Lemma 3.7 by showing that most of the atoms in $\mathcal{P}^{k}$ are small.

Definition 3.5. For $\eta>0, N \geq 0$ we set

$$
\begin{aligned}
V_{\eta, N} & =\left\{\sum_{i, j=0}^{d} a_{i, j} E_{i, j} \in \operatorname{Mat}_{n}(\mathbb{R})|| a_{i, j} \mid \leq\left\{\begin{array}{ll}
\eta e^{-N} & i=1, j \geq 2 \\
\eta & i \geq 2 \text { or } i=j=1
\end{array}\right\},\right. \\
B_{\eta, N} & =\left(I+V_{\eta, N}\right) \cap \mathrm{SL}_{n}(\mathbb{R}) .
\end{aligned}
$$

When $N=0$ we write $V_{\eta}=V_{\eta, 0}$ and $B_{\eta}=B_{\eta, 0}$.

Definition 3.6. Recall that for a lattice $L \subseteq \mathbb{R}^{n}$ we write $h t(L)=h t_{\infty}(L)=\max \left\{\|v\|_{\infty}^{-1} \mid 0 \neq v \in L\right\}$ and set $X^{\geq M}=\{x \in X \mid h t(x)>M\}$. A (finite measurable) partition $\mathcal{P}$ of $X$ is called an $(M, \eta)$ partition if $\mathcal{P}=\left\{P_{0}, P_{1}, \ldots, P_{n}\right\}$ where $P_{0}=X^{\geq M}$ and $P_{i} \subseteq x_{i} B_{\eta}, x_{i} \in \Gamma \backslash G$ for $1 \leq i \leq n$.

The next lemma originally appeared as Lemma 4.5 in [4] for dimension 2, where a slight inaccuracy was corrected in [1]. The generalization to high dimension is straight forward, and for the reader's convenience we add its proof in Appendix A. 
Lemma 3.7 (see [4]). For any $M>1$ there exists some $0<\eta_{0}=\eta_{0}(M, n)$ which satisfies the following. For any $0<\eta \leq \eta_{0}$, an $\left(M, \frac{1}{4} \eta\right)$ partition $\mathcal{P}$ of $X$ and $\kappa \in(0,1), N>0$, there exists some $X^{\prime} \subseteq X \leq M$ such that

(1) $X^{\prime}$ is a union of $S_{1}, \ldots, S_{l} \in \mathcal{P}^{N}$, each of which is contained in a union of at most $C^{\kappa N}$ many balls of the form $g B_{\eta, N}$ with $g \in S_{j}$ for some absolute constant $C$.

(2) $\mu\left(X^{\prime}\right) \geq 1-\mu\left(X^{>M}\right)-\mu^{N}\left(X^{>M}\right) \kappa^{-1}$ for every probability measure $\mu$ on $X$.

Recall that we want to give a lower bound on the entropy where the idea is to show that most of the atoms in $\mathcal{P}^{N}$ have small measure. By Lemma 3.7, most of these atoms are contained in a union of small balls $z B_{\eta, N}$, hence it is important to show that these balls have small measure.

Given $\Lambda \subseteq \Lambda_{q}$ and $(\bar{w}, r) \in \Delta$, the measures $\delta_{\Lambda}^{[\bar{w}, r, q]}$ are averages in the direction of the flow $T$ and in the "horospherical" direction represented by $\Lambda$. While the $T$-average allows us to talk about $T$ invariant measure, the upper bound on the measure $\delta_{\Lambda}^{[\bar{w}, r, q]}\left(z B_{\eta, N}\right)$ will arise from the horospherical direction.

Lemma 3.8. There exists a constant $C(n) \geq 1$ such that the following is true. Given $x \in X$, $0<\eta<\min \left\{\frac{1}{4 n}, \frac{1}{h t(x)}\right\}$ and $q \in \mathbb{N}$, for any $\bar{t} \in \Delta$ and $0 \leq N \leq \ln (q)\left(1+t_{0}-\left(\max _{i \geq 1}\right)\right)$ we have that $\left|\Lambda_{q} a_{q}(\bar{t}) \cap x B_{\eta, N}\right| \leq C(n)\left(\frac{q}{e^{N}}\right)^{n-1}$.

Proof. Writing $x=\Gamma g$ where $g \in \mathrm{SL}_{n}(\mathbb{R})$, an upper bound for $\left|\Lambda_{q} a_{q}(\bar{t}) \cap x B_{\eta, N}\right|$ is given by the number of pairs $(\gamma, \bar{p}) \in \mathrm{SL}_{n}(\mathbb{Z}) \times \Lambda_{q}$ such that

$$
u_{-\bar{p} / q} \gamma g \in a_{q}(\bar{t}) B_{\eta, N}^{-1} \subseteq a_{q}(\bar{t}) B_{2 \eta, N}
$$

where $B_{\eta, N}^{-1} \subseteq B_{2 \eta, N}$ by Lemma A.1.

Letting $\gamma_{i}$ be the rows of $\gamma$, considering the $i$ 'th row of the inclusion above we obtain that

$$
\begin{aligned}
\gamma_{0} z-\sum_{1}^{d} \frac{p_{i}}{q} \gamma_{i} g & \in q^{t_{0}}\left(e_{0}+2 \eta(-1,1) \times 2 \eta\left(-e^{-N}, e^{-N}\right)^{n-1}\right) \\
\gamma_{i} g & =q^{t_{i}}\left(e_{i}+v_{i}\right), v_{i} \in 2 \eta(-1,1)^{n}, \quad i=2, \ldots, n .
\end{aligned}
$$

Suppose that $\gamma_{0}^{\prime} \in \mathbb{Z}^{n}$ such that $\gamma_{1}^{\prime}, \gamma_{2}, \ldots, \gamma_{n}$ form a matrix in $\mathrm{SL}_{n}(\mathbb{Z})$. Writing $\gamma_{0}^{\prime}=\sum_{1}^{n} k_{i} \gamma_{i}, k_{i} \in \mathbb{Z}$, we get that $1=\operatorname{det}\left(\gamma_{1}^{\prime}\left|\gamma_{2}\right| \cdots \mid \gamma_{n}\right)=\operatorname{det}\left(k_{1} \gamma_{1}\left|\gamma_{2}\right| \cdots \mid \gamma_{n}\right)=k_{1}$. Hence, once the $\gamma_{i}, i=2, \ldots, n$ are chosen, any additional solution for equation (3.1) has the form $\gamma_{1} g+\sum_{2}^{n}\left(k_{i}-\frac{p_{i}}{q}\right) \gamma_{i} g$ where $\gamma_{1}$ is also fixed. Denoting by $\pi: \mathbb{R}^{n} \rightarrow \mathbb{R}^{n-1}$ the projection on the hyperplane corresponding to the last $n-1$ coordinate and applying it to equation (3.1) we obtain

$$
\sum_{1}^{d} m_{i} q^{t_{i}}\left(e_{i}+\pi\left(v_{i}\right)\right) \in-q \pi\left(\gamma_{0} g\right)+2 \eta q^{t_{0}} q\left(-e^{-N}, e^{-N}\right)^{n-1}, \quad m_{i}=k_{i} q-p_{i}
$$

To summarize, we wish to bound from above the number of $\gamma_{i} \in \mathbb{Z}^{n}, m_{i} \in \mathbb{Z}$ for $i=2, \ldots, n$ which satisfy equation (3.2) and equation (3.3). Equation (3.2) merely asks how many lattice points $\mathbb{Z}^{n} g$ has in $q^{t_{i}}\left(e_{i}+2 \eta(-1,1)^{n}\right)$. Letting $h=h t_{\infty}\left(\mathbb{Z}^{d+1} g\right)$, we obtain that any set $u+\left[0, \frac{1}{3 h}\right]^{d+1}$ contains at most one point from $\mathbb{Z}^{n} g$, hence $q^{t_{i}}\left(e_{i}+2 \eta(-1,1)^{n}\right)$ contains at most $\left\lceil 12 h \eta q^{t_{i}}\right\rceil^{n} \leq$ $\left\lceil 12 q^{t_{i}}\right\rceil^{n} \leq\left(2^{4} q^{t_{i}}\right)^{n}$ such points. For equation (3.3), we first multiply by the diagonal matrix $a=$ $\operatorname{diag}\left(q^{-t_{2}}, \ldots, q^{-t_{n}}\right)$ to reduce to the problem of counting the lattice points of $L=\operatorname{span}_{\mathbb{Z}}\left\{e_{i}+\pi\left(v_{i}\right) q^{t_{i}} a\right\}$ in $-q \pi\left(\gamma_{0} z\right) a+2 \eta q^{t_{1}} \frac{q}{e^{N}} \prod_{2}^{n}\left(-q^{-t_{i}}, q^{-t_{i}}\right)$. The lattice $L$ is $\mathbb{Z}^{d}\left(I+a^{-1} g_{\eta} a\right)$ where, $g_{\eta} \in M a t_{n}(\mathbb{R}),\left\|g_{\eta}\right\|_{\infty} \leq$ 
EQUIDISTRIBUTION OF DIVERGENT ORBITS OF THE DIAGONAL GROUP IN THE SPACE OF LATTICES 10

$2 \eta<\frac{1}{2 n}$. We shall see in Lemma 3.9 that it implies that $h^{\prime}=h t_{\infty}(L) \leq 2$. It follows that equation (3.3) has at most $\prod_{2}^{n}\left\lceil 12 h^{\prime} \eta q^{t_{1}-t_{i}} \frac{q}{e^{N}}\right\rceil \leq \prod_{2}^{n}\left(2^{6} q^{t_{1}-t_{i}} \frac{q}{e^{N}}\right)$ solutions, where we used the fact that $q^{t_{1}-t_{i}} \frac{q}{e^{N}} \geq 1$. Thus, the total number of solutions is bounded by

$$
\prod_{2}^{n}\left(2^{4} q^{t_{i}}\right)^{n}\left(2^{6} q^{t_{1}-t_{i}} \frac{q}{e^{N}}\right)=C(n)\left(\frac{q}{e^{N}}\right)^{n-1}
$$

Lemma 3.9. Let $g \in M a t_{d}(\mathbb{R})$ with $\|g\|_{\infty}<\frac{1}{2 d}$ and $t_{1}, \ldots, t_{d} \in \mathbb{R}$. Then $h t_{\infty}\left(\mathbb{Z}^{d} \cdot(I+a(\bar{t}) g a(-\bar{t}))\right) \leq$ 2 .

Proof. We note first that $\left\|g^{m}\right\|_{\infty} \leq d^{m-1}\|g\|_{\infty}^{m} \leq \frac{1}{d}\left(\frac{d}{2 d}\right)^{m}$, so that $(I+g)^{-1}=\sum_{0}^{\infty}(-g)^{m}$ is well defined. It follows that $a(\bar{t})(I+g) a(-\bar{t})$ is invertible, hence $\mathbb{Z}^{d} \cdot(I+a(\bar{t}) g a(-\bar{t}))$ is indeed a lattice in $\mathbb{R}^{d}$.

Let $\bar{k}=\left(k_{1}, \ldots, k_{d}\right) \in \mathbb{Z}^{d}$ such that $\|\bar{k}(I+a(\bar{t}) g a(-\bar{t}))\|_{\infty}<\frac{1}{2}$. Computing the $i$-th coordinate produces

$$
\frac{1}{2}>\left|k_{i}+\sum_{1}^{d} k_{j} e^{t_{j}-t_{i}} g_{j, i}\right| \geq\left|k_{i}\right|-\frac{1}{2 d} \sum_{1}^{d}\left|k_{j}\right| e^{t_{j}-t_{i}} .
$$

If $k_{i} \neq 0$, i.e. $\left|k_{i}\right| \geq 1$, then $\left|k_{i}\right| \leq 2\left(\left|k_{i}\right|-\frac{1}{2}\right)<\frac{1}{d} \sum_{j=1}^{d}\left|k_{j}\right| e^{t_{j}-t_{i}}$. Thus there must be some $j=j(i)$ such that $\left|k_{i}\right|<\left|k_{j}\right| e^{t_{j}-t_{i}}$. Consider the directed graph on $\left\{i|| k_{i} \mid \neq 0\right\}$, with an edge $i \rightarrow j$ if $\left|k_{i}\right|<\left|k_{j}\right| e^{t_{j}-t_{i}}$. As we have just seen, each vertex has an outgoing edge, so the graph must contain a directed cycle. Moreover, the defining condition of the edges imply that the graph is transitive, and hence it must has a self loop at some vertex $i$. This is a contradiction since otherwise $\left|k_{i}\right|<\left|k_{i}\right| e^{t_{i}-t_{i}}=\left|k_{i}\right|$. We conclude that $\bar{k}=\overline{0}$, or equivalently $h t_{\infty}\left(\mathbb{Z}^{d} \cdot(I+a(\bar{t}) g a(-\bar{t}))\right) \leq 2$.

3.3. Proof of Theorem 1.4. We now combine all of the results so far to provide a lower bound on the entropy of partial weak star limits $\delta_{\Lambda}^{[\bar{w}, r, q]}$ (which are $a_{q}(\vec{v})$-invariant where $\vec{v}=\frac{1}{n}(1-n, 1, \ldots, 1)$ ). Recall that if a probability measure $\mu$ is a weak star limit of a sequence of probability measures $\mu_{i}$, then for any $\varepsilon>0$ there is some $M>0$ such that $\mu_{i}\left(X^{>M}\right)<\varepsilon$ for all $i$ big enough. We say that the sequence $\mu_{i}$ doesn't exhibit escape of mass if every partial weak limit is a probability measure. The next lemma shows that if $\Lambda \subseteq \Lambda_{q}$ is big, and there is no escape of mass, then we get a good lower bound on the entropy.

Lemma 3.10. Let $\mathcal{P}$ be any $(M, \eta)$-partition with $\eta$ small enough (as a function of $M, n$ ). Let $\Lambda \subseteq \Lambda_{q},(\bar{w}, r) \in E(\Delta)$ such that $q^{(n-1)(1-r)} \leq|\Lambda|$ and $r \ln (q)>1$. Then

$$
\frac{1}{m} H_{\delta_{\Lambda}^{[\bar{w}, r, q]}}\left(\mathcal{P}_{m}\right) \geq\left[(n-1)-\frac{1}{r}\left((n-1)-\frac{\ln |\Lambda|}{\ln (q)}\right)\right]-O\left(\sqrt{\delta_{\Lambda}^{[\bar{w}, r, q]}\left(X^{>M}\right)}+\delta_{\Lambda a_{q}(\bar{w})}\left(X^{>M / e}\right)+\frac{m \ln |\mathcal{P}|}{r \ln (q)}\right) .
$$

Proof. Assume that $\eta$ is small enough and satisfies the conditions of Lemma 3.7 and Lemma 3.8 for all $z \in X^{\leq M}$.

First we move from the continuous measure $\delta_{\Lambda}^{[\bar{w}, r, q]}$ to its discrete version defined by $\delta_{\Lambda}^{\{\bar{v}, r, q\}}:=$ $\frac{1}{[r \ln (q)\rfloor} \sum_{k=0}^{\lfloor r \ln (q)\rfloor-1} \delta_{\Lambda a_{q}(\bar{v}) a(k \vec{v})}$. We have

$\delta_{\Lambda}^{[\bar{w}, r, q]}=\frac{\lfloor\ln (q) r\rfloor}{\ln (q) r} \cdot\left[\int_{0}^{1} \delta_{\Lambda a(x \vec{v})}^{\{\bar{w}, r, q\}} \mathrm{dx}\right]+\frac{\ln (q) r-\lfloor\ln (q) r\rfloor}{\ln (q) r} \cdot\left[\frac{1}{\ln (q) r-\lfloor\ln (q) r\rfloor} \int_{\lfloor\ln (q) r\rfloor}^{\ln (q) r} \delta_{\Lambda a_{q}(\bar{w}) a(x \vec{v})} \mathrm{dx}\right]$. 
EQUIDISTRIBUTION OF DIVERGENT ORBITS OF THE DIAGONAL GROUP IN THE SPACE OF LATTICES 11

Applying Lemma 3.4 we get that

$$
\begin{aligned}
\frac{1}{m} H_{\delta_{\Lambda}^{[\bar{w}, r, q]}}\left(\mathcal{P}^{m}\right) & \geq \frac{1}{m}\left(1-\frac{1}{\ln (q) r}\right) \int_{0}^{1} H_{\delta_{\Lambda a(x, \bar{v})}^{\{(\bar{v}, q\}}}\left(\mathcal{P}^{m}\right) \mathrm{dx} \\
& \geq\left(1-\frac{1}{\ln (q) r}\right)\left[\frac{1}{\lfloor r \ln (q)\rfloor} \int_{0}^{1} H_{\delta_{\Lambda a_{q}(\bar{w}) a(x \vec{v})}}\left(\mathcal{P}^{\lfloor r \ln (q)\rfloor}\right) \mathrm{dx}-\frac{m \ln |\mathcal{P}|}{\lfloor r \ln (q)\rfloor}\right] .
\end{aligned}
$$

Let $X^{\prime}=X_{N, \kappa, M}^{\prime}$ be the set defined in Lemma 3.7 where $N=\lfloor r \ln (q)\rfloor$. If $P \in \mathcal{P}^{N}$ and $P \subseteq X^{\prime}$, then $P \subseteq \bigcup_{1}^{C_{1}^{\kappa N}} g_{i} B_{\eta, N}$ with $g_{i} \in X^{\prime} \subseteq X^{\leq M}$, and hence by Lemma 3.8 we have that $\left|\Lambda_{q} a(\bar{t}) \cap \Gamma g_{i} B_{\eta, N}\right| \leq C_{2} e^{(n-1)(\ln (q)-\lfloor r \ln (q)\rfloor)}$ for any $\bar{t} \in \ln (q) \Delta$, where $C_{1}, C_{2}$ depend only on the dimension $n$. Set $C=\max \left\{1, C_{1}, C_{2} e^{n-1}\right\}$, and note that $C_{2} e^{(n-1)(\ln (q)-\lfloor r \ln (q)\rfloor)} \leq C q^{(n-1)(1-r)}$.

We now have that

$$
\begin{aligned}
H_{\delta_{\Lambda a(\bar{t})}}\left(\mathcal{P}^{N}\right) & =-\sum_{P \in \mathcal{P}_{N}} \delta_{\Lambda a(\bar{t})}(P) \ln \left(\delta_{\Lambda a(\bar{t})}(P)\right) \geq-\sum_{P \in \mathcal{P}_{N} \cap X^{\prime}} \delta_{\Lambda a(\bar{t})}(P) \ln \left(\delta_{\Lambda a(\bar{t})}(P)\right) \\
& \geq-\sum_{P \in \mathcal{P}_{N} \cap X^{\prime}} \delta_{\Lambda a(\bar{t})}(P) \ln \left(C^{\kappa N+1} \frac{q^{(n-1)(1-r)}}{|\Lambda|}\right)=-\delta_{\Lambda a(\bar{t})}\left(X^{\prime}\right) \ln \left(C^{\kappa N+1} \frac{q^{(n-1)(1-r)}}{|\Lambda|}\right) \\
& \geq-(\kappa N+1) \ln (C)+\delta_{\Lambda a(\bar{t})}\left(X^{\prime}\right) \ln \left(\frac{|\Lambda|}{q^{(n-1)(1-r)}}\right),
\end{aligned}
$$

and dividing by $N \leq r \ln (q)$ we obtain

$$
\frac{1}{N} H_{\delta_{\Lambda a(\bar{t})}}\left(\mathcal{P}^{N}\right) \geq \delta_{\Lambda a(\bar{t})}\left(X^{\prime}\right)\left[(n-1)-\frac{1}{r}\left((n-1)-\frac{\ln |\Lambda|}{\ln (q)}\right)\right]-\left(\kappa+\frac{1}{N}\right) \ln (C) .
$$

Note that $(n-1)(1-r) \leq \frac{\ln |\Lambda|}{\ln (q)} \leq n-1$ implies that $0 \leq\left[(n-1)-\frac{1}{r}\left((n-1)-\frac{\ln |\Lambda|}{\ln (q)}\right)\right] \leq n-1$.

Recall from Lemma 3.7 that $\delta_{\Lambda a(\bar{t})}\left(X^{\prime}\right) \geq 1-\delta_{\Lambda a(\bar{t})}\left(X^{>M}\right)-\delta_{\Lambda a(\bar{t})}^{N}\left(X^{>M}\right) \kappa^{-1}$. Applying this inequality with $a(\bar{t})=a_{q}(\bar{w}) a(x \vec{v}), 0 \leq x \leq 1$ and integrating over $x$ we obtain that

$\int_{0}^{1} \delta_{\Lambda a_{q}(\bar{w}) a(x \vec{v})}^{N}\left(X^{>M}\right) \mathrm{dx}=\frac{1}{\lfloor r \ln (q)\rfloor} \int_{0}^{\lfloor r \ln (q)\rfloor} \delta_{\Lambda a_{q}(\bar{w}) a(x \vec{v})}\left(X^{>M}\right) \mathrm{dx} \leq \delta_{\Lambda}^{[\bar{w}, r, q]}\left(X^{>M}\right)+\frac{2}{r \ln (q)}$ and additionally we have that $\delta_{\Lambda a_{q}(\bar{w}) a(x \vec{v})}\left(X^{>M}\right)<\delta_{\Lambda a_{q}(\bar{w})}\left(X^{>M / e}\right)$, so

$$
\int_{0}^{1} \delta_{\Lambda a_{q}(\bar{w}) a(x \vec{v})}\left(X^{\prime}\right) \mathrm{dx} \geq 1-\delta_{\Lambda a_{q}(\bar{w})}\left(X^{>M / e}\right)-\delta_{\Lambda}^{[\bar{w}, r, q]}\left(X^{>M}\right) \kappa^{-1}-\frac{2}{r \ln (q)} .
$$

Combining equation (3.4) equation (3.5) and equation (3.6) we obtain that $\frac{1}{m} H_{\delta_{\Lambda}^{[\bar{w}, r, q]}}\left(\mathcal{P}^{m}\right) \geq\left[(n-1)-\frac{1}{r}\left((n-1)-\frac{\ln |\Lambda|}{\ln (q)}\right)\right]-O\left(\delta_{\Lambda}^{[\bar{w}, r, q]}\left(X^{>M}\right) \kappa^{-1}+\delta_{\Lambda a_{q}(\bar{w})}\left(X^{>e M}\right)+\frac{m \ln |\mathcal{P}|}{r \ln (q)}+\kappa\right)$.

Setting $\kappa=\sqrt{\delta_{\Lambda}^{[\bar{w}, r, q]}\left(X^{>M}\right)}$ if it is nonzero and otherwise $\kappa=\frac{1}{r \ln (q)}$ proves the lemma.

Next we generalize the lemma above to averages over larger sets than just for interval in $\Delta_{q}$ defined by $(\bar{w}, r)$.

Definition 3.11. For $s>0$ we set $\Delta_{s}=\left\{\bar{t} \in \Delta|| t_{i}-t_{j} \mid \leq s \forall i, j \geq 2\right\}$. 
EQUIDISTRIBUTION OF DIVERGENT ORBITS OF THE DIAGONAL GROUP IN THE SPACE OF LATTICES 12

The set $\Delta_{s}$ should be thought of as a tube neighborhood of the line corresponding to $(\overline{0}, 1) \in E(\Delta)$ (in figure 2.1 it is the line from the top vertex to the middle of the triangle). Moreover, thinking of $\Delta_{s}$ as a union of lines $(\bar{w}, r) \in E(\Delta)$, we can present $\mu^{\ln (q) \Delta_{s}}$ as an integral over $\mu^{[\bar{w}, r, q]}$ for any probability measure $\mu$ on $X$. To give a lower bound on these intervals lengths note that for $\bar{t} \in \Delta_{s}$ we have that $\bar{t}+r \vec{v} \in \Delta_{s}$ if and only if $\bar{t}+r \vec{v} \in \Delta$. Thus the conditions on $r$ are that for every $i \geq 2$ we have

$$
t_{i}+\frac{r}{n} \geq 0 \geq-\sum_{2}^{n}\left(t_{j}+\frac{r}{n}\right)=t_{1}+r \frac{(1-n)}{n} \geq\left(t_{i}+\frac{r}{n}\right)-1 .
$$

Equivalently, $r$ is in the interval $\left[-\min _{i \geq 2} n t_{i}, 1+t_{1}-\max _{i \geq 2} t_{i}\right]$. Shifting $\bar{t}$ to $\bar{t}-\left(\min _{i \geq 2} n t_{i}\right) \vec{v}$, we may assume that $\min _{i \geq 2} t_{i}=0$ and that the interval length is $1+t_{1}-\max _{i \geq 2} t_{i}$. Since $\bar{t} \in \Delta_{s}$, we further get that $t_{i} \leq s$ for all $i \geq 2$ and $t_{1}=-\sum_{2}^{n} t_{i} \geq(2-n) s$, so the interval's length is at least $1+(1-n) s$. This leads to the following definition.

Definition 3.12. Recall that $\lambda$ is a fixed Haar measure on $A \cong \mathbb{R}_{0}^{n}$. For $s>0$ define $\nu_{s}$ to be a probability measure on $E(\Delta)$ such that

$$
\left.\frac{1}{\lambda\left(\Delta_{s}\right)} \cdot \lambda\right|_{\Delta_{s}}=\int\left[\frac{1}{r} \int_{0}^{r} \delta_{\bar{w}+t \vec{v}} \mathrm{dt}\right] \mathrm{d} \nu_{s}(\bar{w}, r) .
$$

More over we can choose such $\nu_{s}$ which satisfies $r\left(\nu_{s}\right):=\inf \left\{r \mid(\bar{w}, r) \in \operatorname{supp}\left(\nu_{s}\right)\right\}=\max \{0,1+(1-n) s\}$.

We can now generalize Lemma 3.10.

Theorem 3.13. Fix some $s>0$. For any choice of $\Lambda_{q}^{\prime} \subseteq \Lambda_{q}$, any weak star limit $\mu$ of $\delta_{\Lambda_{q}^{\prime}}^{\ln (q) \Delta_{s}}$ is A-invariant. Suppose in addition that

(1) The sequences $\delta_{\Lambda_{q}^{\prime}}^{\ln (q) \Delta_{s}}=\int \delta_{\Lambda_{q}^{\prime}}^{[\bar{w}, r, q]} \mathrm{d} \nu_{s}$ and $\int \delta_{\Lambda_{q}^{\prime} a_{q}(\bar{w})} \mathrm{d} \nu_{s}$ do not exhibit escape of mass, and

(2) we have $\ell=\liminf _{q \rightarrow \infty} \frac{\ln \left|\Lambda_{q}^{\prime}\right|}{\ln (q)}>0$.

If $\ell=n-1$, then $h_{\mu}(T)=n-1$. If $\ell<n-1$, but $1>(n-1)$ s then $h_{\mu}(T) \geq(n-1)\left(1-\frac{1-\ell /(n-1)}{1-(n-1) s}\right)$.

Proof. By the assumption on the lack of escape of mass, any weak star partial limit of $\delta_{\Lambda_{q}^{\prime}}^{\ln (q) \Delta_{s}}$ is a probability measure, and by the structure of $\Delta_{s}$, it is easily seen to be $A$-invariant.

In order to apply Lemma 3.10, we need to show that most of $\delta_{\Lambda}^{[\bar{w}, r, q]}, \delta_{\Lambda a_{q}(\bar{w})}$ for $(\bar{w}, r) \in \operatorname{supp}\left(\nu_{s}\right)$ do not exhibit escape of mass, and have that $r\left(\nu_{s}\right)>0$. As this is not true in general, we restrict to a submeasure.

By condition (1) we can choose $M$ big enough so that $\delta_{\Lambda_{q}^{\prime}}^{\ln (q) \Delta_{s}^{\prime}}\left(X^{>M}\right), \int \delta_{\Lambda_{q}^{\prime} a_{q}(\bar{w})}\left(X^{>M / e}\right)<\varepsilon$ for all $q$ large enough. Moreover, we can choose $r_{\varepsilon} \geq r\left(\nu_{s}\right)$ with $r_{\varepsilon}>0$ so that

$$
\Omega=\Omega(q, \varepsilon)=\left\{(\bar{w}, r) \mid \delta_{\Lambda_{q}^{\prime}}^{[\bar{w}, r, q]}\left(X^{>M}\right) \leq \sqrt{\varepsilon}, \delta_{\Lambda_{q}^{\prime} a_{q}(\bar{w})}\left(X^{>M / e}\right) \leq \sqrt{\varepsilon}, r \geq r_{\varepsilon}\right\}
$$

satisfies $\nu_{s}(\Omega) \geq 1-3 \sqrt{\varepsilon}$. We set $\nu_{s, \varepsilon}=\left.\frac{1}{\nu(\Omega)} \nu_{s}\right|_{\Omega}$ and $\nu_{s, \varepsilon}^{\prime}=\left.\frac{1}{\nu\left(\Omega^{c}\right)} \nu_{s}\right|_{\Omega^{c}}$ so that $\nu_{s}=\nu_{s}(\Omega) \cdot \nu_{s, \varepsilon}+$ $\left(1-\nu_{s}(\Omega)\right) \nu_{s, \varepsilon}^{\prime}$.

Choose an $(M, \eta)$ partition $\mathcal{P}$ where $\mu(\partial P)=0$ for all $P \in \mathcal{P}$ (there are such partitions for arbitrarily large $M$ and any small enough $\eta>0)$. For such a partition we have that $\frac{1}{m} H_{\mu}\left(\mathcal{P}^{m}\right) \geq$ 
EQUIDISTRIBUTION OF DIVERGENT ORBITS OF THE DIAGONAL GROUP IN THE SPACE OF LATTICES 13 $\frac{1}{m} \limsup H_{\delta_{\Lambda_{q}^{\prime}}^{\ln (q) \Delta_{s}}}\left(\mathcal{P}^{m}\right)$ for any $m \in \mathbb{N}$. Using Lemma 3.4 twice, once from $\delta_{\Lambda_{q}^{\prime}}^{\ln (q) \Delta_{s}}=\int \delta_{\Lambda_{q}^{\prime}}^{[\bar{w}, r, q]} \mathrm{d} \nu_{s}$ to $\delta_{\Lambda_{q}^{\prime}, \varepsilon}^{\nu_{s}}:=\int \delta_{\Lambda_{q}^{\prime}}^{[\bar{w}, r, q]} \mathrm{d} \nu_{s, \varepsilon}$ and a second time to $\delta_{\Lambda_{q}^{\prime}}^{[\bar{w}, r, q]}$, we get that

$$
H_{\delta_{\Lambda_{q}^{\prime}}^{\ln (q) \Delta_{s}}}\left(\mathcal{P}^{m}\right) \geq \nu(\Omega) H_{\delta_{\Lambda_{q}^{\prime}, \varepsilon}^{\nu_{s}}}\left(\mathcal{P}^{m}\right) \geq(1-3 \sqrt{\varepsilon}) \int H_{\delta_{\left.\Lambda_{q}^{\prime}, r, q\right)}^{(\bar{w}}}\left(\mathcal{P}^{m}\right) \mathrm{d} \nu_{s, \varepsilon}(\bar{w}, r) .
$$

Using Lemma 3.10 we conclude that

$$
\begin{aligned}
\frac{1}{m} H_{\mu}\left(\mathcal{P}^{m}\right) & \geq(1-3 \sqrt{\varepsilon}) \limsup _{q \rightarrow \infty}\left[(n-1)-\frac{1}{r_{\varepsilon}}\left((n-1)-\frac{\ln \left|\Lambda_{q}^{\prime}\right|}{\ln (q)}\right)-O\left(2 \sqrt{\varepsilon}+\frac{m \ln |\mathcal{P}|}{r_{\varepsilon} \ln (q)}\right)\right] \\
& \geq(1-3 \sqrt{\varepsilon})\left[(n-1)-\frac{(n-1)-\ell}{r_{\varepsilon}}-O(2 \sqrt{\varepsilon})\right] .
\end{aligned}
$$

Assume first that $r\left(\nu_{s}\right)=1-(n-1) s>0$. Then $\frac{1}{m} H_{\mu}\left(\mathcal{P}^{m}\right) \geq(1-3 \sqrt{\varepsilon})\left[(n-1)-\frac{(n-1)-\ell}{1-d s}-O(2 \sqrt{\varepsilon})\right] \rightarrow$ $(n-1)\left(1-\frac{1-\ell /(n-1)}{1-(n-1) s}\right)$ as $\varepsilon \rightarrow 0$.

If $\ell=(n-1)$, then regardless of whether $r\left(\nu_{s}\right)>0$, we have that $\frac{1}{m} H_{\mu}\left(\mathcal{P}^{m}\right) \geq(1-3 \sqrt{\varepsilon})[(n-1)-O(2 \sqrt{\varepsilon})] \rightarrow$ $(n-1)$ as $\varepsilon \rightarrow 0$, thus completing the proof.

There are two natural settings in which we can apply Theorem 3.13. The first one is when $\Lambda_{q}^{\prime}=\Lambda_{q}$ and then $\liminf _{q \rightarrow \infty} \frac{\ln \left|\Lambda_{q}^{\prime}\right|}{\ln (q)}=n-1$ so we only need to worry about the escape of mass condition. We shall deal with this setting in the next section.

The second setting is when the $\delta_{\Lambda_{q}^{\prime}}^{\ln (q) \Delta_{s}}, \int \delta_{\Lambda_{q}^{\prime} a_{q}(\bar{w})} \mathrm{d} \nu_{s}$ are supported in some fixed compact set $K$, hence condition 1 is automatically satisfied, and any partial weak limit $\mu$ is also compactly supported probability measure. Thus, if $\Lambda_{q}^{\prime}$ is big enough, then $\mu$ has positive entropy. The classification of such measures for $n \geq 3$ was done in [2] by Einsiedler, Katok and Lindenstrauss.

Theorem 3.14. (see [2]) Let $\mu$ be an A-invariant and ergodic probability measure on $X$ with positive entropy with respect to some nontrivial element in $A$. Then $\mu$ is an algebraic measure and is not compactly supported.

From this we can prove Theorem 1.4.

Proof of Theorem 1.4. Fix a compact set $K \subseteq X_{n}$. For $s>0$ we have that $\Lambda_{q, K}=\left\{x \in \Lambda_{q} \mid x \cdot(\ln (q) \Delta) \subseteq K\right\} \subseteq$ $\Lambda_{q, K, s}:=\left\{x \in \Lambda_{q} \mid x \cdot\left(\ln (q) \Delta_{s}\right) \subseteq K\right\}$. By Theorem 3.13 we get that if $\lim _{i \rightarrow \infty} \frac{\ln \left|\Lambda_{q_{i}, K, s}\right|}{\ln \left(q_{i}\right)}=\ell>0$ for any subsequence, then any partial limit $\mu$ of $\delta_{\Lambda_{q_{i}}^{\prime}}^{\ln \left(q_{i}\right) \Delta_{s}}$ is an $A$-invariant probability measure with $h_{\mu}(T) \geq(n-1)\left(1-\frac{1-\ell /(n-1)}{1-(n-1) s}\right)$. In particular, choosing $s$ small enough we get a positive entropy, hence $\mu$ is not bounded in contradiction to Theorem 3.14. We conclude that $\lim _{q \rightarrow \infty} \frac{\ln \left|\Lambda_{q_{i}, K, s}\right|}{\ln (q)}=0$, hence $\left|\Lambda_{q, K}\right|=O\left(q^{\varepsilon}\right)$ for any $\varepsilon>0$.

3.4. No escape of mass and ergodicity. In this section we show that for any $(\bar{w}, r) \in \Delta$, the measures $\delta_{\Lambda_{q}}^{[\bar{w}, r, q]}, \delta_{\Lambda_{q} a_{q}(\bar{w})}$ do not exhibit escape of mass (i.e. when averaging over all the elements in $\Lambda_{q}$ ). Hence condition (1) in Theorem 3.13 is satisfied. Since $\frac{\ln \left|\Lambda_{q}\right|}{\ln (q)}=(n-1) \frac{\ln (\varphi(q))}{\ln (q)} \rightarrow(n-1)$, we obtain that any partial weak limit of $\delta_{\Lambda_{q}}^{\Delta_{s}}$ has maximal entropy and therefore must be the Haar 
measure. As we shall see, the argument for the lack of escape of mass boils down to equidistribution of $(\mathbb{Z} / q \mathbb{Z})^{\times}$in $\mathbb{Z} / q \mathbb{Z}$, and we begin with this result. The results in this section were proved in [1] for $n=2$, and the proofs here are straight forward generalizations to higher dimension.

Definition 3.15. For $N \in \mathbb{N}$ we set $\omega(N)$ to be the number of distinct prime factors of $N$.

Lemma 3.16. Let $N \in \mathbb{N}$ and $0 \leq \alpha \leq 1$. Then ||$\{1 \leq m \leq \alpha N:(m, N)=1\}|-\alpha \varphi(N)| \leq 2^{\omega(N)}$.

Proof. For $P \in \mathbb{N}$ set $U_{P}=P \mathbb{Z} \cap[1, \ldots, \alpha N]$, so that $\left|U_{p}\right|=\left\lfloor\frac{\alpha N}{P}\right\rfloor$. We want to find $U_{1} \backslash \bigcup_{p} U_{p}$ where $p$ runs over the prime divisors of $N$. Letting $\mu(P)$ be the Möbius function we obtain that

$$
\left|U_{1} \backslash \bigcup_{p} U_{p}\right|=\sum_{P \mid N} \mu(P)\left|U_{P}\right|=\alpha N \sum_{P \mid N} \frac{\mu(P)}{P}+\sum_{P \mid N} \mu(P)\left[\left|U_{P}\right|-\frac{\alpha N}{P}\right] .
$$

The lemma is proved by noting that $N \sum_{P \mid N} \frac{\mu(P)}{P}=\varphi(N)$ and that

$$
\left|\sum_{P \mid N} \mu(P)\left[\left|U_{P}\right|-\alpha \frac{N}{P}\right]\right| \leq \sum_{P \mid N}|\mu(P)| \leq 2^{\omega(N)} .
$$

The next lemma shows that sequence of the form $\delta_{\Lambda_{q} a(\bar{t})}$ do not exhibit escape of mass under suitable conditions on $\bar{t}$.

Lemma 3.17 (No escape of mass). Fix some $q \in \mathbb{N}, M>1$ and $\bar{t} \in \ln (q) \Delta$ where $\ln (q) \geq$ $2 \omega(q)+\max _{i \geq 2} t_{i}-t_{1}$. Then

$$
\left|\left\{\bar{p} \in \Lambda_{q} \mid \Gamma u_{\bar{p} / q} a(\bar{t}) \in X^{\geq M}\right\}\right| \leq \frac{(4 \varphi(q))^{n-1}}{M^{n}}=\frac{4^{n-1}}{M^{n}}\left|\Lambda_{q}\right|,
$$

or equivalently $\left(\delta_{\Lambda_{q} a(\bar{t})}\right)\left(X^{\geq M}\right) \leq \frac{4^{n-1}}{M^{n}}$.

Proof. We say that $\bar{p} \in \Lambda_{q}$ is bad if $\Gamma u_{\bar{p} / q} a(\bar{t}) \in X^{\geq M}$, i.e. there exists $\overline{0} \neq(m, \bar{k}) \in \mathbb{Z}^{n}$ such that $\left\|(m, \bar{k}) u_{\bar{p} / q} a(\bar{t})\right\|_{\infty} \leq \frac{1}{M}$ or equivalently $|m| \leq \frac{1}{M e^{t_{1}}}$ and $\left|m p_{i}+q k_{i}\right| \leq \frac{q}{M e^{t_{i}}}$ for $i=2, \ldots, n$. Clearly, we may assume that $m \geq 0$ and actually even $m \geq 1$, since otherwise for each $i \geq 2$ we have $1>\frac{1}{M} \geq\left|\left(m \frac{p_{i}}{q}+k_{i}\right) e^{t_{i}}\right|=\left|k_{i}\right| e^{t_{i}} \geq\left|k_{i}\right|$ and therefore $k_{i}=0$ (because $t_{i} \geq 0$ for $i \geq 2$ ) contradiction.

We say that $\bar{p}$ is $m$-bad if there exist some $\bar{n}$ such that $\left|m p_{i}+q k_{i}\right| \leq \frac{q}{M e^{t_{i}}}$ for $i=2, \ldots, n$.

Fixing $1 \leq m \leq \frac{1}{M e^{t_{1}}}$, we count the number of $m$-bad $\bar{p}$ for each such $m$. Letting $d_{m}=\operatorname{gcd}(q, m)$ and writing $q=\tilde{q} d_{m}, m=\tilde{m} d_{m}$, we get that

$$
\left|\tilde{q} k_{i}+\tilde{m} p_{i}\right| \leq \frac{\tilde{q}}{e^{t_{i} M}} .
$$

We consider equation (3.7) as a counting problem over $\mathbb{Z} / \tilde{q} \mathbb{Z}$. Note first that $\bar{t} \in \ln (q) \Delta$ implies that $t_{1} \geq-\ln (q) \frac{n-1}{n}$, so that $d_{m} \leq m<q^{\frac{n-1}{n}}$ and therefore $\tilde{q}>q^{\frac{1}{n}}>1$. Since $(q, p)=1$ we also have that $(\tilde{q}, p)=1$ and hence $(\tilde{q}, \tilde{m} p)=1$. Consider the map $\pi:(\mathbb{Z} / q \mathbb{Z})^{\times} \rightarrow(\mathbb{Z} / \tilde{q} \mathbb{Z})^{\times}$and let $A_{i}=\left\{[a] \in(\mathbb{Z} / \tilde{q} \mathbb{Z})^{\times}|| a \mid \leq \frac{\tilde{q}}{e^{t_{i} M}}\right\}$. For this fixed $m$, the $m$-bad $\bar{p}$ 's are exactly $\prod_{1}^{k} \pi^{-1}\left(\tilde{m}^{-1} A_{i}\right)$, hence there are at most $\prod_{2}^{n}\left(\left|A_{i}\right| \cdot|\operatorname{ker}(\pi)|\right) \operatorname{such} \bar{p}$. Since $\pi$ is surjective we obtain that $|\operatorname{ker}(\pi)|=\frac{\varphi(q)}{\varphi(\tilde{q})}$ and by the previous lemma we get that $\left|A_{i}\right| \leq 2\left(\frac{1}{e^{t_{i} M}} \varphi(\tilde{q})+2^{\omega(\tilde{q})}\right)$. 
EQUIDISTRIBUTION OF DIVERGENT ORBITS OF THE DIAGONAL GROUP IN THE SPACE OF LATTICES 15

We claim that $2^{\omega(\tilde{q})} \leq \frac{1}{e^{t_{i} M}} \varphi(\tilde{q})$. Assuming this claim, the total number of $m$-bad $p$ 's is at most $\prod_{2}^{n}\left|A_{i}\right| \cdot|\operatorname{ker}(\pi)| \leq \prod_{2}^{n}\left(\frac{4}{e^{t_{i} M}} \varphi(q)\right)=e^{t_{1}}\left(\frac{4 \varphi(q)}{M}\right)^{n-1}$. Since there are $\left\lfloor\frac{1}{M e^{t_{1}}}\right\rfloor$ such $m$, a union bound shows that the number of bad $p$ is at most $\frac{(4 \varphi(q))^{n-1}}{M^{n}}$. Thus, to complete the proof we need only to show that $2^{\omega(\tilde{q})} \leq \frac{1}{e^{t_{i} M}} \varphi(\tilde{q})$ for $i \geq 2$ and $\bar{t} \in \ln (q) \Delta$ such that $\ln (q) \geq 2 \omega(q)+\max _{i \geq 2} t_{i}-t_{1}$.

For any integer with prime decomposition $N=\prod r_{i}^{e_{i}}$ we have that

$$
\varphi(N)=\prod r_{i}^{e_{i}}\left(1-\frac{1}{r_{i}}\right) \geq \frac{1}{e^{\omega(N)}} \prod r_{i}^{e_{i}}=\frac{N}{e^{\omega(N)}} .
$$

The claim now follows from

$$
\frac{2^{\omega(\tilde{q})}}{\varphi(\tilde{q})} \leq \frac{e^{\omega(\tilde{q})}}{e^{-\omega(\tilde{q})} \frac{q}{d_{m}}}=d_{m} e^{2 \omega(\tilde{q})-\ln (q)} \leq \frac{1}{M e^{t_{1}}} \cdot e^{2 \omega(q)-\ln (q)} \leq \frac{1}{M e^{t_{i}}} .
$$

The condition $\ln (q) \geq 2 \omega(q)+\max _{i \geq 2} t_{i}-t_{1}$ in the lemma means that $\bar{t}$ is a bit far from the faces of $\Delta$ which do not contain $\overline{0}$ (these are the dashed lines in figure 2.1). Since $\omega(q)=o(\ln (q)$ ), this is a very weak condition which we use in the next lemma.

Lemma 3.18. For any $(\bar{w}, r) \in E(\Delta)$ and $q \in \mathbb{N}$ large enough so that $\frac{1}{r} \frac{2 \omega(q)}{\ln (q)} \leq \min \left\{1, \frac{4^{n-1}}{M^{n}}\right\}$ we have that $\delta_{\Lambda_{q}}^{[\bar{w}, r, q]}\left(X^{\geq M}\right), \delta_{\Lambda_{q} a_{q}(\bar{w})}\left(X^{\geq M}\right) \leq\left(\frac{4}{M}\right)^{n}$.

Proof. For $s \in \mathbb{R}$ we denote $\bar{w}^{(s)}=\bar{w}+s \vec{v}$ and note that for $r, s \in \mathbb{R}$ we have that

$$
\max _{i \geq 2} w_{i}^{(s)}-w_{1}^{(s)}=(s-r)+\max _{i \geq 2} w_{i}^{(r)}-w_{1}^{(r)} .
$$

The assumption that $\bar{w}^{(r)} \in \Delta$ implies that $\max _{i \geq 2} w_{i}^{(r)}-w_{1}^{(r)} \leq 1$. Applying Lemma 3.17 we conclude that if $r-s \geq \frac{2 \omega(q)}{\ln (q)}$, then $\delta_{\Lambda_{q} a_{q}\left(\bar{w}^{(s)}\right)}\left(X^{\geq M}\right) \leq \frac{4^{n-1}}{M^{n}}$. By assumption, this is true for $s=0$ and therefore $\delta_{\Lambda_{q} a_{q}(\bar{w})}\left(X^{\geq M}\right) \leq \frac{4^{n-1}}{M^{n}}$. The proof is completed by noting that

$$
\delta_{\Lambda_{q}}^{[\bar{w}, r, q]}\left(X^{\geq M}\right)=\frac{1}{r} \int_{0}^{r} \delta_{\Lambda_{q} a_{q}\left(\bar{w}^{(s)}\right)}\left(X^{\geq M}\right) \mathrm{ds} \leq \frac{1}{r}\left(r \frac{4^{n-1}}{M^{n}}+\frac{2 \omega(q)}{\ln (q)}\right) \leq\left(\frac{4}{M}\right)^{n} .
$$

We can now use this lack of escape of mass and Theorem 3.13 in order to prove Theorem 1.2.

Proof of Theorem 1.2. We first prove (3).

By Lemma 3.18 and Theorem 3.13 we get that $\delta_{\Lambda_{q}}^{\ln (q) \Delta} \stackrel{w^{*}}{\longrightarrow} \mu_{\text {Haar }}$, hence part (3) follows by applying Corollary 2.6.

(3) $\Rightarrow(2)$ : Suppose that $\left|\Lambda_{q}^{\prime}\right| \geq \alpha\left|\Lambda_{q}\right|$ for some $\alpha>0$ and write

$$
\delta_{\Lambda_{q}}^{\ln (q) \Delta_{\text {full }}}=\frac{\left|\Lambda_{q}^{\prime}\right|}{\left|\Lambda_{q}\right|} \delta_{\Lambda_{q}^{\prime}}^{\ln (q) \Delta_{\text {full }}}+\frac{\left|\Lambda_{q} \backslash \Lambda_{q}^{\prime}\right|}{\left|\Lambda_{q}\right|} \delta_{\Lambda_{q} \backslash \Lambda_{q}^{\prime}}^{\ln (q) \Delta_{\text {full }}} .
$$

Suppose that $\mu_{1}$ is a partial weak limit of $\delta_{\Lambda_{q}^{\prime}}^{\ln (q) \Delta_{\text {full }}}$, and note in particular that it is $T$-invariant. By restricting to a subsequence we may assume that all the terms above converge and by taking the limit and applying part (3) we obtain that $\mu_{\text {Haar }}=\alpha^{\prime} \mu_{1}+\left(1-\alpha^{\prime}\right) \mu_{2}$ where $\alpha^{\prime} \geq \alpha>0$. If 
$\alpha^{\prime}=1$, then we are done. Otherwise we must have that $\mu_{2}$ is also $T$-invariant and both $\mu_{1}, \mu_{2}$ are probability measures. Since $\mu_{\text {Haar }}$ is $T$-ergodic, it is an extreme point in the set of $T$-invariant probability measures, implying that $\mu_{1}=\mu_{2}=\mu_{\text {Haar }}$. It follows that $\delta_{\Lambda_{q}^{\prime}}^{\ln (q) \Delta_{\text {full }}}$ converges to the Haar measure as well.

$(2) \Rightarrow(1)$ : Let $\left\{f_{i}\right\}_{1}^{\infty}$ be a dense sequence of compactly supported continuous function in $C_{c}(X)$. For each $m, q \in \mathbb{N}$ define

$$
\Lambda_{q, m}=\left\{x \in \Lambda_{q}\left|\max _{1 \leq i \leq m}\right|\left(\delta_{x}^{\ln (q) \Delta_{\text {full }}}-\mu_{\text {Haar }}\right)\left(f_{i}\right) \mid<\frac{1}{m}\right\} .
$$

We claim that $\lim _{q \rightarrow \infty} \frac{\left|\Lambda_{q, m}\right|}{\left|\Lambda_{q}\right|}=1$ for any fixed $n$. Otherwise, find some $1 \leq i \leq m, \epsilon \in\{ \pm 1\}$ and $\alpha>0$ such that

$$
V_{q}:=\left\{x \in \Lambda_{q} \mid \epsilon\left(\delta_{x}^{\ln (q) \Delta_{\text {full }}}-\mu_{\text {Haar }}\right)\left(f_{i}\right) \geq \frac{1}{m}\right\}
$$

satisfy $\frac{\left|V_{q_{j}}\right|}{\left|\Lambda_{q}\right|} \geq \alpha$ for some subsequence $q_{j}$. By part (2) we get that $\delta_{V_{q}}^{\ln (q) \Delta_{\text {full }}} \rightarrow \mu_{\text {Haar }}$ while $\delta_{V_{q}}^{\ln (q) \Delta_{f u l l}}\left(f_{i}\right) \nrightarrow \nrightarrow \mu_{\text {Haar }}\left(f_{i}\right)$ - contradiction. Define $m(q)=\max \left\{m \mid \frac{\left|\Lambda_{q, m}\right|}{\left|\Lambda_{q}\right|} \geq 1-\frac{1}{m}\right\}$ and set $\Lambda_{q}^{\prime}=\Lambda_{q, m(q)}$. By the claim that we just proved we get that $m(q) \stackrel{q \rightarrow \infty}{\longrightarrow} \infty$, and therefore $\frac{\left|\Lambda_{q}^{\prime}\right|}{\left|\Lambda_{q}\right|} \geq$ $1-\frac{1}{m(q)} \rightarrow 1$. Furthermore, for each $i \leq m$ we have that $\left|\left(\delta_{x}^{\ln (q) \Delta_{\text {full }}}-\mu_{\text {Haar }}\right)\left(f_{i}\right)\right|<\frac{1}{m}$ whenever $x \in \Lambda_{q}^{\prime}$ and $m(q) \geq m$. We conclude that $\delta_{x^{(q)}}^{\ln (q) \Delta_{\text {full }}}\left(f_{i}\right) \rightarrow \mu_{\text {Haar }}\left(f_{i}\right)$ for any choice of $x^{(q)} \in \Lambda_{q}^{\prime}$, and since $\left\{f_{i}\right\}_{1}^{\infty}$ is dense we have that $\delta_{x(q)}^{\ln (q) \Delta_{\text {full }}} \stackrel{w^{*}}{\longrightarrow} \mu_{\text {Haar }}$ and we are done.

\section{Appendix A. Proof of Lemma 3.7}

Before proving Lemma 3.7, we give a few result about small balls in $X_{n}$ which we need in the proof. Recall (from Definition 3.5) that for $\eta>0, N \geq 0$ we set

$$
\begin{aligned}
V_{\eta, N} & =\left\{\sum_{i, j=1}^{n} a_{i, j} E_{i, j}|| a_{i, j} \mid \leq\left\{\begin{array}{ll}
\eta e^{-N} & i=1, j \geq 2 \\
\eta & i \geq 2 \text { or } i=j=1
\end{array}\right\},\right. \\
B_{\eta, N} & =\left(I+V_{\eta, N}\right) \cap \mathrm{SL}_{n}(\mathbb{R}),
\end{aligned}
$$

and denote $V_{\eta}=V_{\eta, 0}$ and $B_{\eta}=B_{\eta, 0}$.

It is easy to check that $V_{\eta, N} \cdot V_{\eta^{\prime}, N} \subseteq V_{\eta \eta^{\prime} n, N}$ and $V_{\eta, N}+V_{\eta^{\prime}, N} \subseteq V_{\eta+\eta^{\prime}, N}$. In particular we get the following two simple results.

Lemma A.1. Let $I+W \in B_{\eta, N}$ where $\eta<\frac{1}{2 n}$. Then $(I+W)^{-1} \in I-W+V_{\eta^{2} n, N}$ and in particular $\left(B_{\eta, N}\right)^{-1} \subseteq B_{\frac{3}{2} \eta, N}$.

Proof. Given any $W \in V_{\eta, N}$ we have that $W^{k} \in V_{\eta(\eta n)^{k}, N}$ and hence $\sum_{2}^{\infty}(-W)^{k} \in \sum_{1}^{\infty} V_{\eta(\eta n) \frac{1}{2^{k}, N}}=$ $V_{\eta(\eta n), N}$ is well defined. It then follows that $(I+W)^{-1}=I-W+\sum_{2}^{\infty}(-W)^{k} \in I-W+V_{\eta(\eta n), N}$.

Lemma A.2. Let $g, h \in \Gamma \backslash G$ such that $h \in g B_{\eta, N}$ for $\eta<\frac{1}{2 n}$. Then $g B_{\eta, N} \subseteq h B_{4 \eta, N}$.

Proof. Write $h=g \tilde{h}$ for some $\tilde{h} \in B_{\eta, N}$. Then $g B_{\eta, N}=h \tilde{h}^{-1} B_{\eta, N} \subseteq h B_{\eta, N}^{-1} B_{\eta, N} \subseteq y B_{\frac{3}{2} \eta, N} B_{\eta, N}$. The claim now follows from the fact that $V_{\frac{3}{2} \eta, N}+V_{\eta, N}+V_{\frac{3}{2} \eta, N} V_{\eta, N} \subseteq V_{4 \eta, N}$. 
EQUIDISTRIBUTION OF DIVERGENT ORBITS OF THE DIAGONAL GROUP IN THE SPACE OF LATTICES 17

The following lemma shows that we can cover an $(\eta, N)$ ball by $\left(\frac{\eta}{R}, N\right)$ balls where the number of small balls needed is bounded as a function of $R$.

Lemma A.3. Let $R \in \mathbb{R}_{>1}$ and $0<\eta<\frac{1}{6 n R}$. There exists less than $(8 R)^{n^{2}}$ elements $\gamma_{i} \in B_{\eta, N}$ such that $B_{\eta, N} \subseteq \bigcup \gamma_{i} B_{\frac{\eta}{R}, N}$. If $S \subseteq B_{\eta, N}$ is given, then we can choose $\gamma_{i} \in S$ such that $S \subseteq \bigcup \gamma_{i} B_{\frac{\eta}{R}, N}$.

Proof. The cover argument is obvious if we work in the additive world, i.e. in $V_{\bar{r}}$ instead, so we start there and write $V_{\eta, N}=\bigcup_{1}^{K}\left(v_{i}+V_{\frac{\eta}{4 R}, N}\right)$ where $K=(4\lceil R\rceil)^{n^{2}} \leq(8 R)^{n^{2}}$. Suppose that $W_{1}, W_{2} \in v_{i}+V_{\frac{\eta}{4 R}, N}$, and we wish to show that $\left(I+W_{1}\right)^{-1}\left(I+W_{2}\right)$ are in a small ball $B_{\frac{\eta}{R}, N}$. that

If $W_{1}, W_{2} \in v_{i}+V_{\frac{\eta}{4 R}, N}$, then setting $W_{1}^{\prime}=\sum_{2}^{\infty}(-W)^{k}$, which is in $V_{\eta^{2} n, N}$ by Lemma A.1, we get

$$
\begin{aligned}
\left(I+W_{1}\right)^{-1}\left(I+W_{2}\right) & =\left(I-W_{1}+W_{1}^{\prime}\right)\left(I+W_{2}\right)=I+\left(W_{2}-W_{1}\right)-W_{1} W_{2}+W_{1}^{\prime}+W_{1}^{\prime} W_{2} \\
& \in I+V_{\frac{\eta}{2 R}, N}+V_{\eta^{2} n, N}+V_{\eta^{2} n, N}+V_{\eta^{3} n^{2}, N} \subseteq I+V_{\frac{\eta}{2 R}, N}+V_{3 \eta^{2} n, N} \subseteq I+V_{\frac{\eta}{R}, N} .
\end{aligned}
$$

For each $1 \leq i \leq K$ choose $\gamma_{i} \in \mathrm{SL}_{n}(\mathbb{R}) \cap\left(I+v_{i}+V_{\frac{\eta}{4 R}, N}\right)$ if this set is not empty, so by the argument above, any $I+W \in \mathrm{SL}_{n}(\mathbb{R}) \cap\left(I+V_{\frac{\eta}{R}, N}\right)$ satisfies $\gamma_{i}^{-1}(I+W) \in B_{\frac{\eta}{R}, N}$, and we conclude that $B_{\eta, R} \subseteq \bigcup_{1}^{K} \gamma_{i} B_{\frac{\eta}{R}, N}$.

If $S \subseteq B_{\eta, N}$ is given, then choosing $\gamma_{i} \in S \cap\left(I+v_{i}+V_{\frac{\eta}{4 r}}, N\right)$ will satisfy the requirements of the lemma.

We now use these lemmas in order to prove Lemma 3.7.

Proof of Lemma 3.7. Choose $0<\eta_{0}(M, n)<\frac{1}{6 n}$ to be small enough so that the map $g \mapsto x g$ from $B_{\eta_{0} e} \rightarrow \Gamma \backslash G$ is injective for all $x \in X^{\leq M}$ and let $\mathcal{P}=\left\{P_{0}, \ldots, P_{\ell}\right\}$ be an $(M, \eta)$ partition, $\eta<\eta_{0}(M)$.

Consider the function $f(x)=\frac{1}{N} \sum_{0}^{N-1} 1_{X>M}\left(T^{i} x\right)$ and note that this function is constant on each $P \in \mathcal{P}_{N}$.

Setting $X^{\prime}=X^{\leq M} \cap\{x: f(x) \leq \kappa\}$, we obtain that

$$
\begin{aligned}
1 & \leq \mu\left(X^{>M}\right)+\mu(\{f(x)>\kappa\})+\mu\left(X^{\prime}\right) \leq \mu\left(X^{>M}\right)+\kappa^{-1} \int f(x) \mathrm{d} \mu+\mu\left(X^{\prime}\right) \\
& =\mu\left(X^{>M}\right)+\mu^{N}\left(X^{>M}\right) \kappa^{-1}+\mu\left(X^{\prime}\right)
\end{aligned}
$$

thus proving part (3) in the theorem.

Suppose that $S \in \mathcal{P}_{N}, S \subseteq X^{\prime}$ and let $V_{m}=\left|\left\{0 \leq i \leq m \mid T^{i}(S) \subseteq X^{>M}\right\}\right|$. Let $C$ be the constant from Lemma A.3 For $R=e$. We claim that $S \subseteq \bigcup_{1}^{C^{\left|V_{m}\right|}} h_{i, m} B_{\eta, m}$ where $h_{i, m} \in S$ for any $0 \leq m \leq N$, and the lemma will follow by taking $m=N-1$. For $m=0$, let $h \in S \subseteq P_{i} \subseteq g_{i} B_{\frac{\eta}{4}}$ for some $i \geq 1$, so by Lemma A.2 we obtain that $S \subseteq h B_{\eta}$, thus proving the case for $m=0$.

Assume that $S \subseteq \bigcup_{i=1}^{C\left|V_{m}\right|} h_{i, m} B_{\eta, m}, h_{i, m} \in S$ for $m<N-1$ and we prove for $m+1$. We set $a=a\left(\frac{1}{n}(1-n, 1, \ldots, 1)\right) \in A$.

- Suppose first that $T^{m+1} S \subseteq X \leq M$ so that $T^{m+1} S \subseteq P_{j} \subseteq g_{j} B_{\frac{\eta}{4}}$ for some $j \geq 1$. This case will be complete if we can show that $S \cap h_{i, m} B_{\eta, m}=S \cap h_{i, m} B_{\eta, m+1}$ for every $i$. Indeed, Lemma A.2 implies that $T^{m+1} S \subseteq h_{i, m} a^{m+1} B_{\eta}$, so if $h_{i, m} g \in S$ with $g \in B_{\eta, m}$, then

$$
\left[h_{i, m} a^{(m+1)}\right] a^{-(m+1)} g a^{(m+1)}=h_{i, m} g a^{(m+1)} \in T^{m+1} S \subseteq y_{i} a^{(m+1)} B_{\eta} .
$$


By the assumption on the injectivity radius and since $a^{-(m+1)} g a^{(m+1)} \in B_{\eta e}$, we conclude that $g \in B_{\eta, m} \cap a^{(m+1)} B_{\eta} a^{-(m+1)}=B_{\eta, m+1}$ which is what we wanted to show.

- Suppose now that $T^{n+1} S \subseteq X^{>M}$. By Lemma A.3, for each $i$ we have that $S \cap h_{i, m} B_{\eta, m} \subseteq$ $\bigcup_{j=1}^{C} \tilde{h}_{i, m}^{(j)} B_{\frac{\eta}{4 e}, m}$. Choose $h_{i, m}^{(j)} \in S \cap \tilde{h}_{i, m}^{(j)} B_{\frac{\eta}{4 e}, m}$ if this set is not empty and otherwise choose $h_{i, m}^{(j)} \in S$ arbitrarily. By Lemma A.2 it follows that $\bigcup_{j=1}^{C} \tilde{h}_{i, m}^{(j)} B_{\frac{\eta}{4 e}, m} \subseteq \bigcup_{j=1}^{C} h_{i, m}^{(j)} B_{\frac{\eta}{e}, m} \subseteq$ $\bigcup_{j=1}^{C} h_{i, m}^{(j)} B_{\eta, m+1}$, which completes the proof.

\section{REFERENCES}

[1] Ofir David and Uri Shapira. Equidistribution of divergent orbits and continued fraction expansion of rationals. arXiv:1707.00427 [math], July 2017. arXiv: 1707.00427.

[2] Manfred Einsiedler, Anatole Katok, and Elon Lindenstrauss. Invariant Measures and the Set of Exceptions to Littlewood's Conjecture. Annals of Mathematics, 164(2):513-560, September 2006.

[3] Manfred Einsiedler and Elon Lindenstrauss. Diagonal actions on locally homogeneous spaces.

[4] Manfred Einsiedler, Elon Lindenstrauss, Philippe Michel, and Akshay Venkatesh. The distribution of closed geodesics on the modular surface, and Duke's theorem. Enseign. Math., 58(3):249-313, 2012.

[5] Manfred Einsiedler, Elon Lindenstrauss, and Ward Thomas. Entropy in ergodic theory and homogeneous dynamics. To appear. Preprint available at http://www.personal.leeds.ac.uk/ mattbw/entropy.

[6] Uri Shapira and C Zheng. Limiting distributions of translates of divergent diagonal orbits. Preprint.

[7] George Tomanov and Barak Weiss. Closed orbits for actions of maximal tori on homogeneous spaces. Duke Math. J., 119(2):367-392, August 2003.

[8] Peter Walters. An Introduction to Ergodic Theory. Springer Science \& Business Media, October 2000.

Department of Mathematics, Technion, Haifa, Israel

E-mail address: ushapira@tx.technion.ac.il

Department of Mathematics, Hebrew University, Jerusalem, Israel

E-mail address: ofir.david@mail.huji.ac.il 